\title{
Highly Excited Motion in Molecules: Saddle-Node Bifurcations and Their Fingerprints in Vibrational Spectra
}

\author{
M. Joyeux \\ Laboratoire de Spectrométrie Physique, Université Joseph Fourier-Grenoble I, BP 87, \\ F-38402 St Martin d'Heres Cedex, France
}

\author{
S. C. Farantos \\ Institute of Electronic Struture and Laser Foundation for Research and Technology-Hellas, Greece, and \\ Department of Chemistry, University of Crete, Iraklion 711 10, Crete, Greece \\ R. Schinke* \\ Max-Planck-Institut für Strömungsforschung, D-37073 Göttingen, Germany
}

Received: August 8, 2001; In Final Form: December 12, 2001

\begin{abstract}
The vibrational motion of highly excited molecules is discussed in terms of exact quantum and classical mechanics calculations, employing global potential energy surfaces, as well as in terms of a spectroscopic Hamiltonian and its semiclassical limit. The main focus is saddle-node bifurcations and their influence on the spectrum. The general features are illustrated by three examples, which despite their quite different intramolecular motions have several aspects in common: $\mathrm{HCP}, \mathrm{HOCl}$, and $\mathrm{HOBr}$. In all three cases a 1:2 Fermi resonance is the ultimate cause of the complications observed in the spectra.
\end{abstract}

\section{Introduction}

Close to the bottom of a potential energy surface (PES), vibrational motion can be well described by normal modes. ${ }^{1}$ The normal mode picture is based on approximating the PES up to second-order about the equilibrium configuration. This leads to a Hamiltonian, which is the sum of uncoupled harmonic oscillator Hamiltonians. For a molecule with $N$ atoms and $3 N$ -6 vibrational degrees of freedom, there are $3 N-6$ normal modes, and each vibrational state can be assigned a set of quantum numbers $\left(v_{1}, v_{2}, \ldots, v_{3 N-6}\right)$. The motion associated with this state consists of $v_{1}$ quanta of excitation in the harmonic oscillator associated with normal mode 1 , plus $v_{2}$ quanta of excitation in harmonic oscillator associated with normal mode 2 , etc. up to mode $v_{3 N-6}$.

However, this simple picture usually is valid only for low energies or, alternatively, for motion confined to small displacements from the equilibrium. As energy increases, the assumptions of the normal mode picture gradually break down. First, the individual oscillators will become more anharmonic, and second, and more importantly, the coupling between the oscillators will steadily increase. One possible consequence is that the normal modes, loosely speaking, vary with energy. For example, an almost pure bend normal mode can acquire a substantial stretch character as energy increases. Therefore, assigning a set of quantum numbers is not sufficient to characterize a quantum state; one also has to identify the type of vibration associated with each particular mode. More critical, however, is the frequent observation that some normal modes can be destroyed while new ones appear. Within classical mechanics, this happens at discontinuities of the classical phase space known as bifurcations. ${ }^{2,3}$ Therefore, the set of basis functions into which the vibrational motion is decomposed varies with increasing energy, and one has to indicate explicitly what basis is used for a given set of $\left(v_{1}, v_{2}, \ldots, v_{3 N-6}\right)$ quantum numbers. At last, the coupling between all the normal modes becomes so strong that the classical motion becomes chaotic ${ }^{3,4}$ and the nodal structure of most of the quantum mechanical wave functions becomes so bizarre that a meaningful assignment is obsolete. The reason for all this to happen is the departure of the molecule from the region of the coordinate space where an expansion of the potential up to second order is appropriate.

The development of vibrational motion from the low-energy regime to high excitations has been studied in great detail, especially in the context of nonlinear dynamics. However, the vast majority of investigations has used either simple, mostly two-mode systems, like the Henon-Heiles Hamiltonian, ${ }^{5,6}$ or Hamiltonians based on only few experimentally observed lowlying vibrational states ${ }^{7-10}$ (see the end of section VI for a short discussion of somewhat more complex systems investigated very recently). Nevertheless, these simple models have been very illuminating in studying the interesting nonlinear motions, which take place at high excitation energies. ${ }^{11}$ Thanks to the advent of more and more powerful computers and efficient ab initio electronic structure methods, ${ }^{12}$ it has become possible to construct potential energy surfaces (PESs) for real molecules, which are global, that is, which encompass the equilibrium as well as configurations far away from equilibrium, including the breaking of one or several bonds. The PES of ozone in the ground electronic state is an illustrative recent example. ${ }^{13} \mathrm{With}$ these PES at hand, an expansion of the potential up to low order is dispensable. 


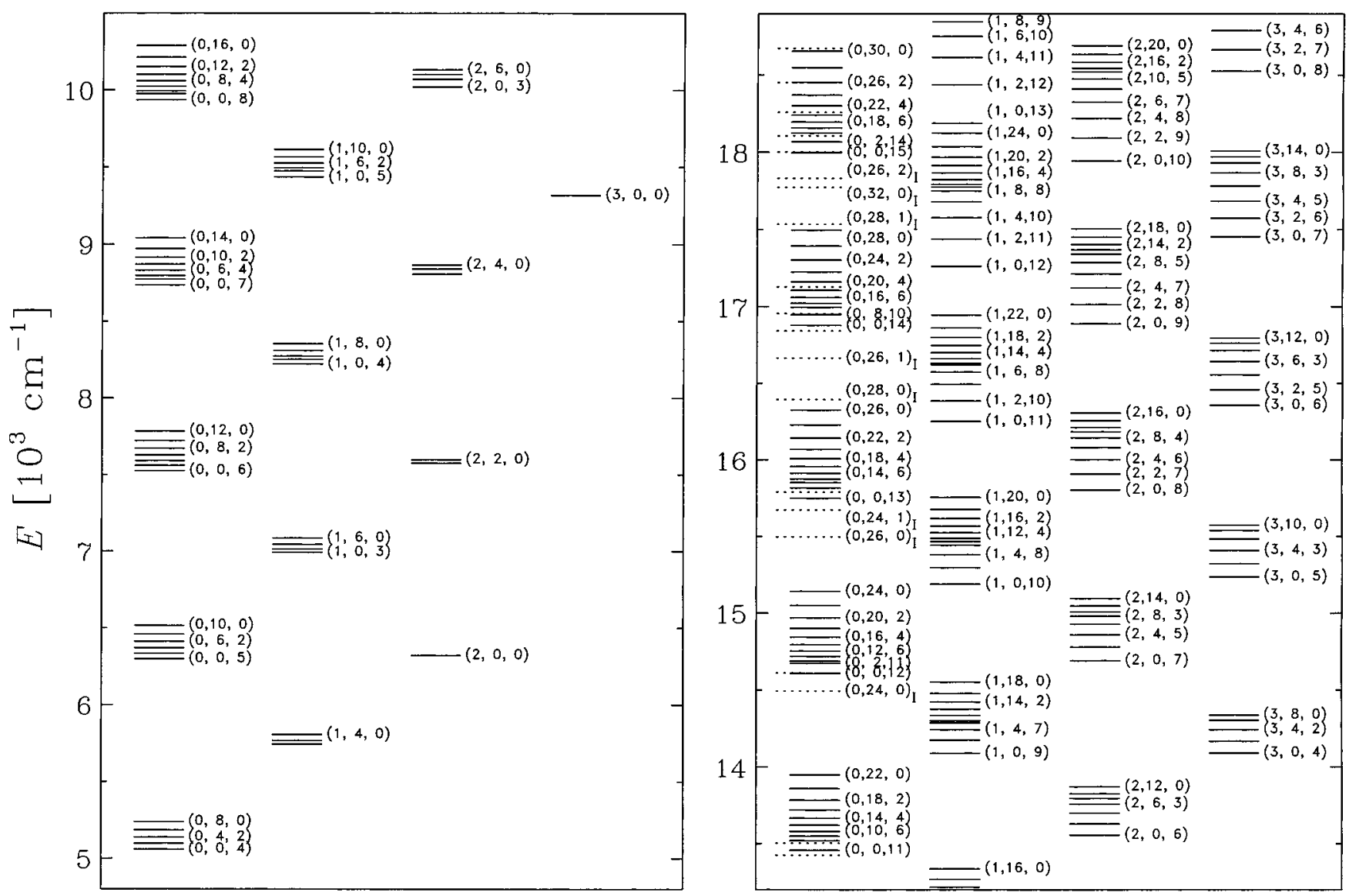

Figure 1. Calculated energy-level spectrum of HCP in two energy regimes. $v_{1}, v_{2}$, and $v_{3}$ are the HC stretch, the bend, and the CP stretch quantum numbers, respectively. The isomerization states born at the saddle-node bifurcation are indicated by the dotted lines. Reprinted, with permission of Annual Review of Physical Chemistry, from ref 19 (copyright 1999).

The achievements in constructing accurate PES are paralleled by advances in calculating hundreds of energy levels by solving exactly the Schrödinger equation for the motion of the atoms on these PES. ${ }^{14-16}$ For a triatomic molecule it is nowadays possible to calculate all bound states and their wave functions up to the dissociation limit. ${ }^{13}$ Inspecting the wave functions shows whether a meaningful assignment is possible and what kind of motion is connected with the quantum numbers or whether the nodal structure is so complex that an assignment is not obvious. ${ }^{17}$

Such complete studies (accurate PES, exact level calculations, and assignment for hundreds of states) have been performed for $\mathrm{HCP}^{18,19}$ and $\mathrm{DCP},{ }^{20} \mathrm{HOCl},{ }^{21-23} \mathrm{HOBr},{ }^{24,25} \mathrm{HCO}^{26,27}$ and DCO, ${ }^{28,29}$ and $\mathrm{HCN} .{ }^{30,31}$ These studies allow us to investigate for real molecules how the vibrational motion, encoded in the spectrum, develops from the bottom of the potential well all the way to the dissociation threshold. Except for the dynamics of HCN, which looks deceptively simple, it turns out, as expected, that bifurcations profoundly affect the spectra. In particular, the spectra of $\mathrm{HCP}, \mathrm{HOCl}, \mathrm{HOBr}$, and $\mathrm{DCO}$ are governed by saddle-node (or tangent) bifurcations, whereas that of DCP is governed by several period-doubling (or pitchfork) bifurcations. ${ }^{32,33}$

To illustrate the complexity of a spectrum at high excitation energies, we compare in Figure 1 for HCP the spectrum in the low-energy region (left panel) with the spectrum at considerably higher energies (right panel). In both cases, the spectrum consists of clusters of levels, so-called polyads (see below), which are organized according to the $\mathrm{HC}$ stretch quantum number $v_{1}=$ $0,1, \ldots$. While in the low-energy portion all polyads are quite compact and well separated, at higher energies they become considerably wider and strongly overlap, which makes the assignment more difficult. The qualitative change of the spectrum is partly related to a saddle-node bifurcation. It is not difficult to surmise how complex the spectrum becomes at even higher energies. Experimentally, ${ }^{34}$ states up to $\sim 25000 \mathrm{~cm}^{-1}$ have been analyzed!

The principal aim of this Feature Article is to give an overview of the basic results of these studies and to emphasize the common framework of the spectra, with the hope that experimental and theoretical spectroscopists understand better the high-energy portions of spectra of other molecules. It must be noted that due to modern spectroscopic methods such as stimulated emission pumping ${ }^{35}$ (SEP), it is possible today to record at least parts of spectra at high energies. ${ }^{36}$ Because of lack of space we will concentrate on saddle-node bifurcations here. They have been much less studied than the normal-tolocal mode bifurcations, ${ }^{2,37-39}$ and they are much less familiar to the spectroscopic community, although both types of bifurcations have several properties in common, especially the more or less abrupt birth of new types of modes in a narrow energy regime.

The most thorough picture about the vibrational dynamics and how it shows up in the spectrum is obtained when we look from different perspectives. The foundation is the global ab initio PES and the exact vibrational eigenstates obtained from the solution of the Schrödinger equation (section II). Viewing each wave function, which in practice needs a lot of patience, reveals how the basic structure of the spectrum changes when energy increases. Additional insight is gained from analyzing the 
classical phase space and how its construction reorganizes with energy (section III). Effective (or resonance or spectroscopic) Hamiltonians, frequently used by spectroscopists to fit experimental data, provide yet supplementary information (section IV). They uncover the primary cause for the bifurcations, namely the nonlinear resonance between two normal modes. Furthermore, they effectively allow a reduction of the number of degrees of freedom by introducing approximate quantum numbers and are a convenient basis for semiclassical treatments.

Sufficient experimental data, which beyond doubt confirm the existence of saddle-node bifurcations, to our knowledge, are available only for $\mathrm{HCP} .{ }^{40} \mathrm{~A}$ thorough comparison between the substantial set of spectroscopic data for $\mathrm{HCP}$ and the theoretical results can be found in refs 18, 19, and 41. Spectroscopic information for $\mathrm{HOCl}$, the other cornerstone of this article, is much sparser. ${ }^{42,43}$ For technical reasons only states with large excitations of the $\mathrm{OH}$ stretch have been analyzed experimentally; although these states are very interesting for investigating the dissociation of $\mathrm{HOCl},{ }^{44-46}$ they exhibit a clear local-mode behavior and therefore are not relevant for the topic of this article. Although $\mathrm{HOBr}$ behaves much like $\mathrm{HOCl}$, there are distinct differences. Because this study is not completely finished yet, we will concentrate the discussion on HCP and $\mathrm{HOCl}$.

\section{Quantum Mechanical Point of View: The Genesis of "Isomerization" and "Dissociation" States}

The purpose of this section is 3-fold: (1) outlining the general approach of exact quantum mechanical calculations on a global PES, (2) summarizing the characteristic features of the molecules the article is basically centered on- $\mathrm{HCP}$, on one hand, and $\mathrm{HOCl}$ and $\mathrm{HOBr}$, on the other-, and (3) presenting the essential observations made in the quantum mechanical studies, which actuated our combined efforts.

The cornerstone of any realistic dynamical investigation of intramolecular motion and the resulting spectrum is a global PES, $V(q)$, where $q$ comprises a complete set of coordinates necessary for describing the molecule. The ab initio construction of a PES is a two-step process. First, the potential energies have to be calculated from first principles by using high-level electronic structure theory at many nuclear geometries. All regions of the coordinate space that are energetically accessible must be covered. This includes, of course, geometries far away from the equilibrium. Usually, for a triatomic molecule without symmetry a thousand points or so are sufficient. Particular regions such as, e.g., isomerization or dissociation barriers may require a higher density of points.

The second step is the fitting of the calculated energies to an analytical expression to be used in the dynamics calculations. ${ }^{47,48}$ It must be stressed that a power series expansion in many cases is not appropriate, because it is not likely to reproduce all the dynamically relevant features with sufficient precision. Fitting a PES over broad coordinate and energy ranges is probably the most tedious, though necessary, task in constructing a PES.

The calculated ${ }^{18}$ PES of HCP is depicted in Figure 2. It has an interesting "peanut"-like shape with the waist occurring where the HC bond is broken and a PH bond begins to be formed. ${ }^{49}$ Needless to say, representing such a shape by a power series expansion around the equilibrium is bound to fail.

The main properties of the PES of $\mathrm{HCP}, \mathrm{HOCl}$, and $\mathrm{HOBr}$ are summarized in Table 1 . HCP is linear at equilibrium. ${ }^{18,49}$ However, the other linear configuration, $\mathrm{CPH}$, is not a local minimum but a saddle, which is about $27000 \mathrm{~cm}^{-1}$ above the $\mathrm{HCP}$ well. Although $\mathrm{CPH}$ is not a real minimum of the PES,

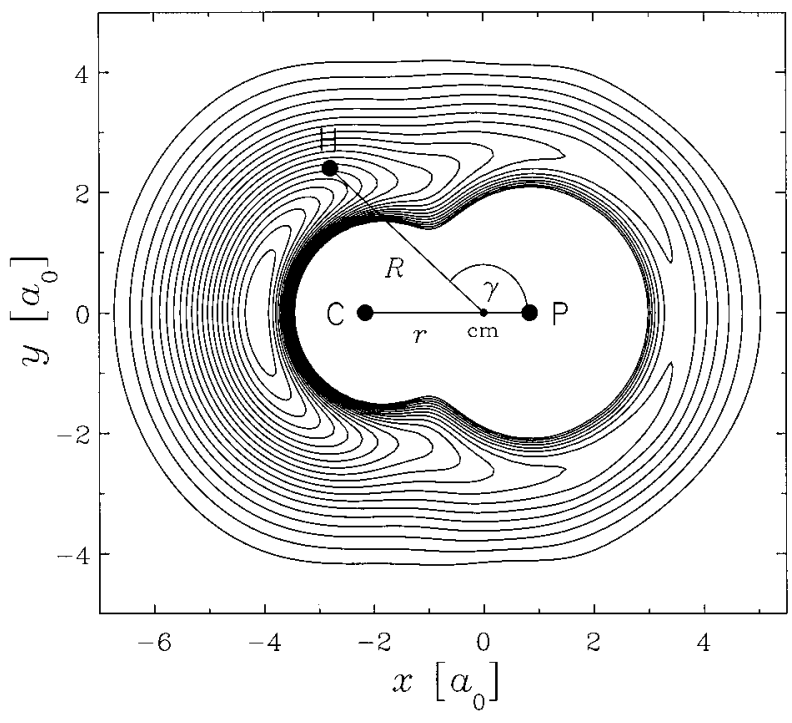

Figure 2. Contour plot of the HCP ground-state potential energy surface. The CP bond distance is fixed. $R, r$, and $\gamma$ are the Jacobi coordinates used in the dynamics calculations. Reprinted, with permission of Annual Review of Physical Chemistry, from ref 19 (copyright 1999).

the $\mathrm{HCP} / \mathrm{CPH}$ system has been investigated as a prototype for an isomerization reaction. Large-angle $\mathrm{H}$-atom bending motion would be a better characterization.

In contrast to $\mathrm{HCP}$, both $\mathrm{HOCl}$ and $\mathrm{HOBr}$ have a bent equilibrium geometry and their isomers, $\mathrm{OClH}$ and $\mathrm{OBrH}$, are true minima of the energy surfaces. ${ }^{21,24,50-53}$ However, except for three states of $\mathrm{HClO}$, all of the levels belonging to the $\mathrm{HClO}$ and $\mathrm{HBrO}$ wells are located above the lowest dissociation threshold. Moreover, the dissociation energies for the HOX $\rightarrow$ $\mathrm{HO}+\mathrm{X}$ reactions (Table 1 ) are much smaller than the energies of the isomerization barriers and therefore the $\mathrm{OClH}$ and $\mathrm{OBrH}$ isomers will be disregarded throughout.

Although their intramolecular dynamics and hence their spectra are quite different, $\mathrm{HCP}, \mathrm{HOCl}$, and $\mathrm{HOBr}$ have something important in common, which allows us to discuss them together: The reaction coordinate is involved in a Fermi resonance ${ }^{54,55}$ with another mode. For the $\mathrm{HCP} \leftrightarrow \mathrm{CPH}$ isomerization the reaction coordinate is obviously the bending angle, while for the $\mathrm{HOX} \rightarrow \mathrm{HO}+\mathrm{X}(\mathrm{X}=\mathrm{Cl}$ and $\mathrm{Br})$ dissociation the reactive coordinate is the OX stretch. The Fermi resonance plays a central role in molecular dynamics whenever there exist two modes, such that twice the fundamental frequency of one mode (which hereafter will be called the "slow" mode) is approximately equal to the frequency of the other mode (to be called the "fast" mode), i.e., $2 \omega_{\mathrm{s}} \approx \omega_{\mathrm{f}}$. Because of this resonance condition, all states that share the same polyad number

$$
P=2 v_{\mathrm{f}}+v_{\mathrm{s}}
$$

where $v_{\mathrm{f}}$ and $v_{\mathrm{s}}$ denote respectively the number of quanta in the fast and slow mode, remain close in energy over broad energy ranges. As a result of this the Fermi resonance, which couples states with the same value of $P$, has a very pronounced influence on the spectrum. In triatomic molecules there exists only one additional mode, which in all three cases, however, remains fairly decoupled from the other two modes over the entire range of energies studied and therefore will be labeled with a "u" (for "uncoupled"). In the remainder of this article, a polyad $\left[v_{\mathrm{u}}, P\right]$ will denote the set of states coupled by the Fermi resonance. 
TABLE 1: Spectroscopic and Computational Information for the Three Molecules Discussed

\begin{tabular}{|c|c|c|c|}
\hline & $\mathrm{HCP}$ & $\mathrm{HOCl}$ & $\mathrm{HOBr}$ \\
\hline lowest feature of PES & $\begin{array}{l}\text { isomerization saddle } \\
\mathrm{HCP} \leftrightarrow \mathrm{CPH} \\
27360 \mathrm{~cm}^{-1}\end{array}$ & $\begin{array}{l}\text { dissociation } \\
\mathrm{HOCl} \rightarrow \mathrm{HO}+\mathrm{Cl} \\
D_{0}=19290 \mathrm{~cm}^{-1}\end{array}$ & $\begin{array}{l}\text { dissociation } \\
\mathrm{HOBr} \rightarrow \mathrm{HO}+\mathrm{Br} \\
D_{0}=17227 \mathrm{~cm}^{-1}\end{array}$ \\
\hline exact quantum & $\begin{array}{l}1000 \text { calculated states } \\
\approx 750 \text { assigned states }\end{array}$ & $\begin{array}{l}827 \text { calculated states } \\
\approx 500 \text { assigned states }\end{array}$ & $\begin{array}{l}706 \text { calculated states } \\
\approx 650 \text { assigned states }\end{array}$ \\
\hline resonance Hamiltonian ${ }^{a}$ & $\begin{array}{l}\text { first } 370 \text { states } \\
E \leq g_{000}+18750 \mathrm{~cm}^{-1} \\
77 \% \text { of the saddle energy } \\
30 \text { quanta in mode s } \\
\Delta_{\text {rms }}=7.7 \mathrm{~cm}^{-1}, N_{\mathrm{p}}=15\end{array}$ & $\begin{array}{l}\text { first } 702 \text { states } \\
E \leq g_{000}+18650 \mathrm{~cm}^{-1} \\
97 \% \text { of } D_{0} \\
38 \text { quanta in mode s } \\
\Delta_{\text {rms }}=5.3 \mathrm{~cm}^{-1}, N_{\mathrm{p}}=28\end{array}$ & $\begin{array}{l}\text { first } 592 \text { states } \\
E \leq g_{000}+16400 \mathrm{~cm}^{-1} \\
95 \% \text { of } D_{0} \\
37 \text { quanta in mode s } \\
\Delta_{\text {rms }}=7.3 \mathrm{~cm}^{-1}, N_{\mathrm{p}}=34\end{array}$ \\
\hline $\begin{array}{l}\text { mode } s \\
\text { mode } f \\
\text { mode u }\end{array}$ & $\begin{array}{l}\text { bend }\left(\omega_{2}=698 \mathrm{~cm}^{-1}\right) \\
\operatorname{CP}\left(\omega_{3}=1301 \mathrm{~cm}^{-1}\right) \\
\operatorname{CH}\left(\omega_{1}=3343 \mathrm{~cm}^{-1}\right)\end{array}$ & $\begin{array}{l}\mathrm{OCl}\left(\omega_{3}=754 \mathrm{~cm}^{-1}\right) \\
\text { bend }\left(\omega_{2}=1259 \mathrm{~cm}^{-1}\right) \\
\mathrm{OH}\left(\omega_{1}=3777 \mathrm{~cm}^{-1}\right)\end{array}$ & $\begin{array}{l}\operatorname{OBr}\left(\omega_{3}=633 \mathrm{~cm}^{-1}\right) \\
\text { bend }\left(\omega_{2}=1183 \mathrm{~cm}^{-1}\right) \\
\operatorname{OH}\left(\omega_{1}=3755 \mathrm{~cm}^{-1}\right)\end{array}$ \\
\hline $\begin{array}{l}\mathrm{SN} \text { bifurcation } \\
\quad \text { for } v_{\mathrm{u}}=0^{b}\end{array}$ & $\begin{array}{l}E=g_{000}+7744 \mathrm{~cm}^{-1} \\
P_{\mathrm{SN}}=12.30\end{array}$ & $\begin{array}{l}E=g_{000}+13135 \mathrm{~cm}^{-1} \\
P_{\mathrm{SN}}=21.76\end{array}$ & $\begin{array}{l}E=g_{000}+5663 \mathrm{~cm}^{-1} \\
P_{\mathrm{SN}}=9.78\end{array}$ \\
\hline
\end{tabular}

${ }^{a} \Delta_{\mathrm{rms}}=$ root-mean-square error, $N_{\mathrm{p}}=$ number of parameters. ${ }^{b} P_{\mathrm{SN}}=$ polyad number at which the saddle-node bifurcation occurs.

The data in Table 1 confirm that for HCP twice the frequency of the bend $\left(2 \omega_{2}=1396 \mathrm{~cm}^{-1}\right)$ is close to the frequency of the $\mathrm{CP}$ stretch $\left(\omega_{3}=1301 \mathrm{~cm}^{-1}\right)$, whereas for $\mathrm{HOCl}$ and $\mathrm{HOBr}$, twice the frequency of the OX stretch $\left(2 \omega_{3}=1508 \mathrm{~cm}^{-1}\right.$ and $2 \omega_{3}=1266 \mathrm{~cm}^{-1}$, respectively) is not too far from the frequency of the bend $\left(\omega_{2}=1259 \mathrm{~cm}^{-1}\right.$ and $\omega_{2}=1183 \mathrm{~cm}^{-1}$, respectively). These coincidences strongly hint to possible couplings due to Fermi resonances and must be taken into consideration early on. As the reader will see below, the Fermi resonance between the reactive coordinate and one of the other coordinates (and therefore the possibility for energy to flow between them) is the principal cause of interesting observations in the spectra. Conversely, no special effects associated with large-amplitude motion in the reactive coordinate are expected or observed, when the reaction coordinate remains decoupled from the other degrees of freedom up to the reaction threshold (this is the case, for example, for the bend degree of freedom in the $\mathrm{HCN} \leftrightarrow \mathrm{CNH}^{31}$ and $\mathrm{DCP} \leftrightarrow \mathrm{CPD}^{20}$ isomerizations).

The comparison between the three molecules can be carried even further. First, the normal mode built on the reactive coordinate is the slow mode in the Fermi resonance. Second, in all three cases $2 \omega_{\mathrm{s}}>\omega_{\mathrm{f}}$ such that, up to moderate energies, the overtone states corresponding to the slow mode are expected at the top of the polyads, whereas those corresponding to the fast mode are expected at the bottom. However, the PES naturally is most anharmonic in the reaction coordinate (slow mode) so that at high energies, closer to the isomerization saddle or the dissociation limit, the ordering of the overtone states must be reversed, that is, the states corresponding to the reactive mode must be at the bottom of the polyads. It is this interplay of the Fermi resonance and the drastic change of the anharmonicity of the PES along the reaction coordinate that leads to the interesting features in the quantum mechanical spectra to be discussed next.

Once the PES is known, it is used, together with an exact expression for the kinetic energy operator, to calculate the rovibrational spectrum by solving the Schrödinger equation. ${ }^{14-16}$ Usually, Jacobi coordinates $R, r$, and $\gamma$ are employed (see Figure 2 for HCP). The main difficulty is to find an appropriate basis set for expanding the wave function. One usually starts with products of one-dimensional basis functions, ${ }^{15,16,56}$ for example, spherical harmonics for angular motions and oscillator wave functions for the stretches. Alternatively, one can exploit more general basis sets, which are defined by discrete points on multidimensional grids (discrete variable representation, $\mathrm{DVR}^{57}$ ).
In any case, to represent highly excited states, a very large number of such primitive basis functions are required, typically several hundred thousand of them for triatomic molecules. This is by far too large for direct diagonalization of the Hamiltonian. The problem can be circumvented by using methods such as filter diagonalization, ${ }^{58,59}$ which are devised for performing very accurate calculations in narrow energy windows, or by reducing the basis sets to a manageable size (less than 10000 or so) through several steps of contraction/truncation schemes. ${ }^{14,60}$ Both methods have been applied in the studies presented below.

As indicated in Table 1, many hundreds of states have been computed for each molecule. The wave function for each state has been visually examined in order to check whether it makes sense to assign quantum numbers to them. It turned out that even close to the isomerization saddle in HCP or the dissociation threshold of $\mathrm{HOCl}$ and $\mathrm{HOBr}$ still many states can be rigorously assigned. ${ }^{18,22,23,25}$ In the case of $\mathrm{HOCl}$ (and probably $\mathrm{HOBr}$ as well) a large number of states located even above the threshold for dissociation (so-called "resonance states", ref 61) can be assigned. ${ }^{23}$ This has, of course, important consequences for the dissociation rates and the applicability of statistical models. ${ }^{62}$ Assigning hundreds of states by analyzing the wave function structure is a tedious undertaking. However, if one wants to understand how the spectrum develops from low to high energies, it is absolutely essential.

After completing the assignment, all the states belonging to the same polyad $\left[v_{\mathrm{u}}, P\right]$ are collected and their wave functions are plotted in the plane spanned by the slow and the fast coordinates, because this is the plane in which the dynamically interesting developments as the energy increases can be seen most clearly. Examples at intermediate energies are displayed in Figure 3 for $\mathrm{HOCl}$. Shown are the $P / 2+1$ (seven) wave functions belonging to polyad $[0,12]$ centered at about 7500 $\mathrm{cm}^{-1}$ above the lowest vibrational state. They evolve in a regular manner from a wave function with $P / 2$ nodes along the (vertical) axis for the fast coordinate at the bottom of the polyad, state $(0,6,0)$, to a wave function with $P$ nodes along the (horizontal) axis for the slow coordinate at the top of the polyad, state $(0$, $0,12)$. The latter one is slightly curved, which manifests noticeable energy flow between the slow and the fast coordinate caused by the Fermi resonance even at these moderate energies. At higher energies, the backbones of these curved wave functions will develop a horseshoe-type structure characteristic for the 2:1 resonance (see below). Despite the curvature, all wave functions unambiguously can be assigned three quantum 

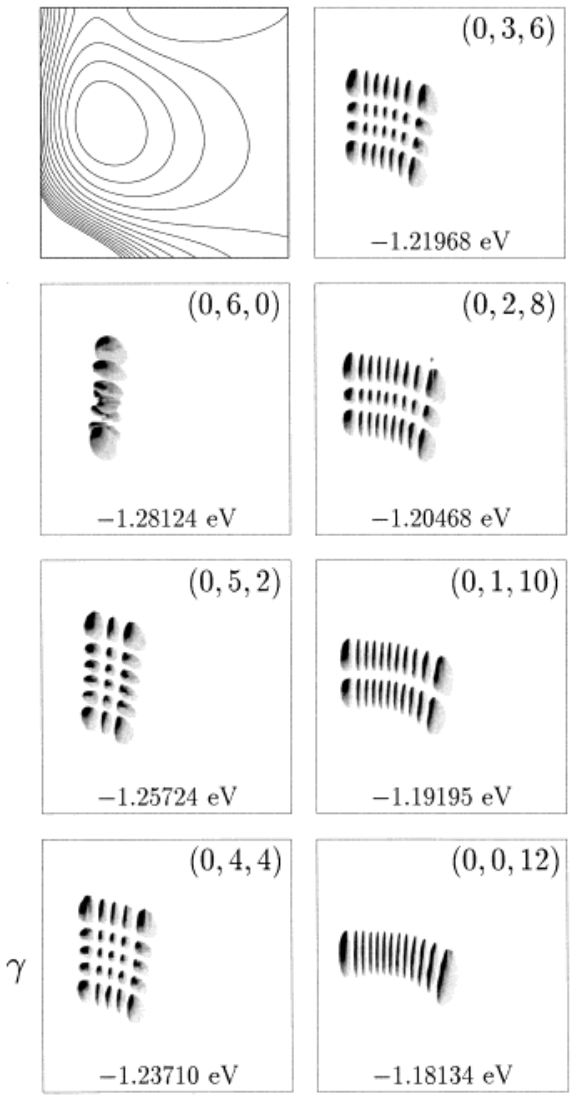

$R$

Figure 3. Wave functions of $\mathrm{HOCl}$ at low energies. $v_{1}, v_{2}$, and $v_{3}$ are the $\mathrm{HO}$ stretch, the bend, and the $\mathrm{OCl}$ stretch quantum numbers, respectively. State $(0,6,0)$ is at the bottom and state $(0,0,12)$ marks the top of the polyad $[0,12]$. Energy normalization is such that $\mathrm{HO}\left(r_{\mathrm{e}}\right)$ $+\mathrm{Cl}$ corresponds to $E=0$. For orientation, the potential energy surface is shown in the upper left panel. Reprinted, with permission of the American Institute of Physics, from ref 23 (copyright 2000).

numbers, with the caveat that the overtones of the slow mode (the top state in each polyad) are no longer associated with motion exclusively along the reactive coordinate but have acquired a nonnegligible contribution from the fast mode. [The quantum numbers for $\mathrm{HOCl}$ and $\mathrm{HOBr}$ are $v_{1}=\mathrm{OH}$ stretch (coordinate $r$ ), $v_{2}=$ bend $(\gamma)$, and $v_{3}=\mathrm{OCl} / \mathrm{OBr}$ stretch $(R)$.] Although the coordinates involved in the Fermi resonance are different for HCP, the wave functions of HCP at intermediate energies are very similar to the wave functions shown in Figure 3 , with the difference that $r$ is the fast coordinate and $\gamma$ the slow one. In contrast to $\mathrm{HOCl}$, the $\mathrm{HCP}$ wave functions are symmetric with respect to the origin of the angular axis. [The quantum numbers for HCP are $v_{1}=\mathrm{HC}$ stretch (coordinate $R$ ), $v_{2}=$ bend $(\gamma)$, and $v_{3}=\mathrm{CP}$ stretch $(r)$.]

Continuing to considerably higher energies, severe changes of the wave function structures become apparent. Figures 4 and 5 depict the complete sets of wave functions for polyad $\left[v_{\mathrm{u}}, P\right]$ $=[0,30]$ of $\mathrm{HOCl}$ and $[0,32]$ of $\mathrm{HCP}$, respectively. They are centered at about $17200 \mathrm{~cm}^{-1}$ for $\mathrm{HOCl}$ and $19500 \mathrm{~cm}^{-1}$ for $\mathrm{HCP}$. The state at the top of the polyad for $\mathrm{HOCl},(0,0,30)$, still displays the horseshoe-shaped wave function discussed above, the horseshoe being, however, more pronounced and narrower than in Figure 3. This means that for $P=30$ the fast coordinate contributes more to the underlying motion than the reactive one, although a naive progression-type assignment would still describe this state as the 30th overtone of the slow reactive mode. The same holds for $\mathrm{HCP}$, too. The assignment of many of the $\mathrm{HOCl}$ states appears mysterious at the first glance. However, systematically following the states from the low-energy part of the spectrum confirms the quoted assignments. For HCP one sees already perturbations from other polyads, e.g., states $(0,32,0)$ and $(0,22,5)$. The perturbations obviously become more pronounced with energy.

More striking, however, are the following observations: (i) The overtones of the fast mode, which were located at the bottom of the polyads (see Figure 3 for $\mathrm{HOCl}$ ), have now moved inside the polyads $[(0,15,0)$ for $\mathrm{HOCl}$ and $(0,0,16)$ for $\mathrm{HCP}]$; (ii) several members of the regular progression are missing $[(0$, $13,4),(0,12,6), \ldots,(0,9,12)$ for $\mathrm{HOCl}$ and $(0,10,11), \ldots,(0$, 4,14 ) for HCP], and (iii) the missing states are replaced, close to the bottom of the polyad, by states with new kinds of wave functions [e.g. $(0,0, x)_{\mathrm{D}(30)}$ for $\mathrm{HOCl}$ and $(0,32,0)_{\mathrm{I}}$ for $\mathrm{HCP}$ ]. Because the latter follow very closely the reaction pathway, they have been termed "isomerization" states for HCP and "dissociation" states for $\mathrm{HOCl}$ and $\mathrm{HOBr}$ in refs $18,19,23,41$, and 63 in order to emphasize their role as precursors of the isomerization and dissociation reactions. It must be emphasized that the new states do not exist in the low-energy regime; they come into existence rather abruptly at intermediate energies.

The existence of the isomerization states for HCP had been first predicted by calculations employing a PES of limited accuracy. ${ }^{64,65}$ Inspired by this prediction, Ishikawa et al. ${ }^{40}$ have searched for these states and indeed found a series of states, which could not be assigned in the way the other levels were assigned. Moreover, these states showed a large anharmonicity and had unusually large rotational $B_{0}$ constants, just the criteria typical for the isomerization states. ${ }^{19}$ The subsequent calculations with the more accurate PES unambiguously confirmed that the levels found by the experimentalists are the predicted isomerization states. ${ }^{18}$

Systematically analyzing the wave functions from the bottom of the well up to high energies clearly shows how the polyad structure changes. However, it does not give a clue why these changes happen. Explanations are provided by classical periodic orbits (PO) and their bifurcations in the classical phase space, which will be the topic of the next section.

Before concluding this section, it is, however, worth mentioning that the existence of the horseshoe-type and the isomerization/dissociation-type states in the same polyad create a problem of assigning these states. For the polyad [0, 30] shown in Figure 4 , for example, one is tempted to describe two different states as the 30th overtone of the reactive mode, two states as the combination with 28 quanta in the reactive mode and 1 quantum in the fast one, and so on. To solve this ambiguity, we could have chosen, as for the distinction of normal and local states, to use square brackets [...] for assigning the isomerization/ dissociation states and parentheses (...) for assigning the states belonging to the normal progressions, which evolve in a regular manner from the low-energy regime. ${ }^{66-68} \mathrm{We}$ instead add either an "I" (for "isomerization") or a "D" (for "dissociation") as an index in order to specify that this state belongs to the new progression, which follows the reaction pathway.

\section{Classical Mechanics Point of View: Periodic Orbits and Saddle-Node Bifurcations}

The purposes of this section are (1) to emphasize the role of stable periodic orbits (POs) as backbones of quantum mechanical wave functions, (2) to emphasize their role in assigning quantum states, and (3) to demonstrate that the birth of the new quantum states, phenomenologically described in section 2 , is related to the existence of saddle-node bifurcations in the classical phase space. 


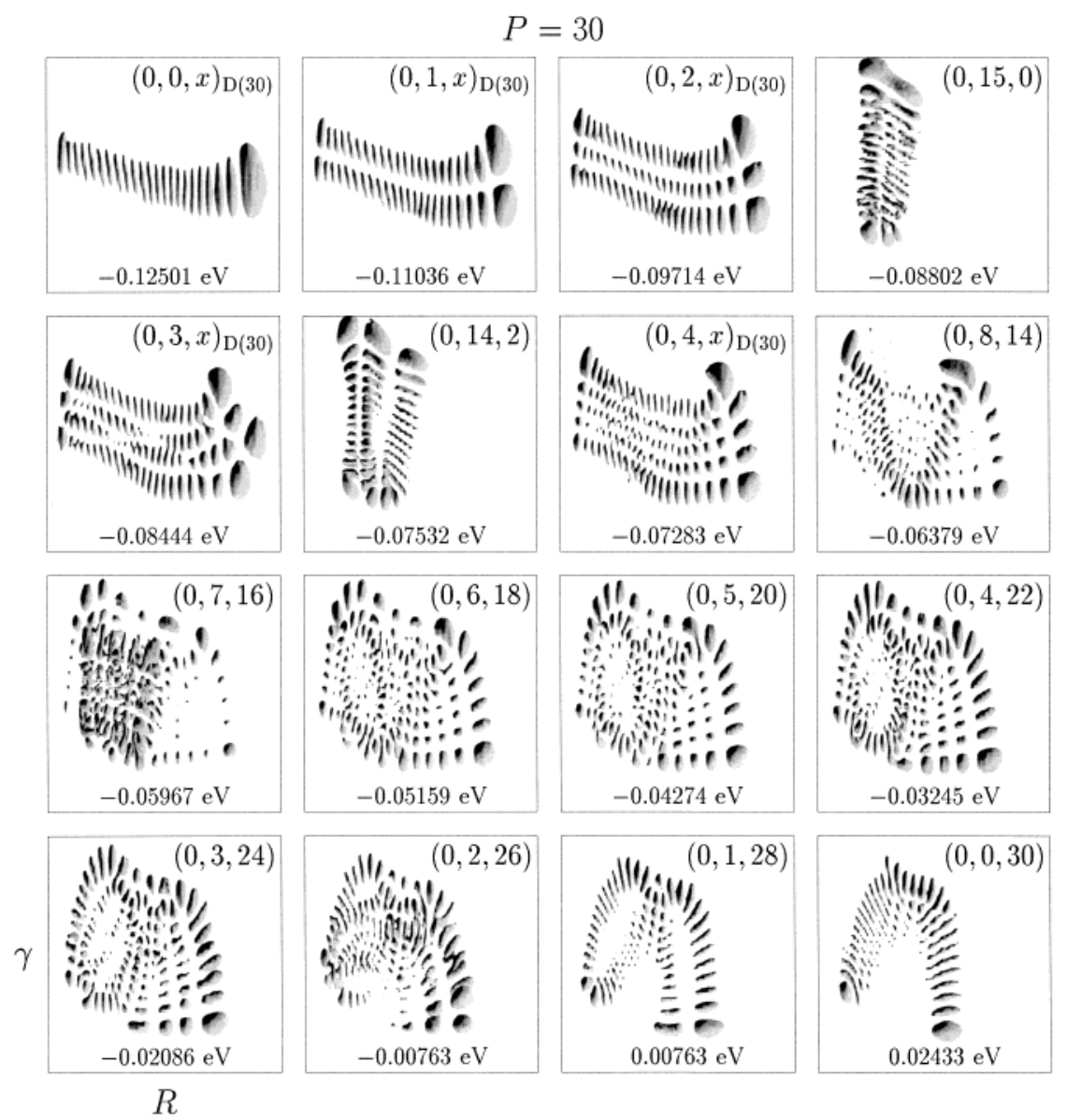

Figure 4. Wave functions for $\mathrm{HOCl}$ in polyad $[0,30]$. Energy normalization is such that $\mathrm{HO}\left(r_{\mathrm{e}}\right)+\mathrm{Cl}$ corresponds to $E=0$. The upper left panel is the bottom of the polyad and the lower right panel marks the top of the polyad. Reprinted, with permission of American Institute of Physics, from ref 23 (copyright 2000).

Periodic orbits, i.e., closed classical trajectories on which the molecule remains for an infinite time,,$^{2,69-71}$ are essential for understanding the structure of the classical phase space. ${ }^{69,72}$ One distinguishes between stable POs and unstable ones. If a trajectory is started close to a stable PO, it remains close to it for all times, even if the trajectory is not periodic. In contrast, trajectories launched in the vicinity of an unstable PO depart exponentially from it. Loosely speaking, each stable PO is at the center of an independent subspace of the total phase space that defines a distinct type of motion, whereas unstable POs separate different subspaces. Finding POs for a system with more than two degrees of freedom is not simple, principally because the strong coupling among the degrees of freedom under normal circumstances introduces large instabilities. Several numerical methods have been proposed for solving this problem. ${ }^{73-75}$ In our work we use shooting methods, which convert the problem of finding POs to an initial value problem: One chooses initial values for a trajectory, integrates Hamilton's equations of motion for a given time, and compares the initial and final positions in the phase space. If the difference is unacceptably large, the initial coordinates and momenta and additionally the integration time are modified.

Just above the minimum of the PES the normal modes and the stable POs coincide. ${ }^{76,77}$ This means that if one puts a small amount of energy in a particular normal mode and lets the system evolve, the energy remains in this mode forever and the trajectory, which the system follows, is a stable PO. Moreover, there exist no other POs beside those obtained by depositing the energy successively in each normal mode. In view of this one-to-one correspondence at very low energies, one is tempted to identify POs with normal modes. However, because of the increasing coupling the dynamics rapidly becomes more complex as the energy increases. Consequently, if one puts more energy in one mode, then part of it is transferred to the other modes and the resulting trajectory is usually not a PO. Nevertheless, in integrable systems or systems close to an integrable one, the fundamental POs, i.e., those found at lower energies, persist to higher energies, even though they may gradually change their shapes as a consequence of the energy transfer between the modes.

The major difference between normal modes and POs concerns their numbers as energy is increased: While, for a triatomic molecule, there are always three normal modes, the number of stable POs can be, as a consequence of bifurcations in particular saddle-node (SN) bifurcations, larger. Saddle-node (or tangent) bifurcations are discontinuities of the classical phase space, where a stable and an unstable PO are created simultaneously. 2,3,32,33,69,72 This is illustrated in Figure 6, which shows the Poincaré surfaces of section of a two-dimensional model for $\mathrm{HOCl}$ at different energies (measured with respect to the energy of the quantum mechanical ground state). ${ }^{63}$ Here, only the two lowest energies are relevant. In these representations POs appear as single points, because the PO crosses the surface only one time during each period. At $12500 \mathrm{~cm}^{-1}$, i.e., just below the first $\mathrm{SN}$ bifurcation, there are only three POs, labeled $[R],[\gamma]$, and $[r]$ (the latter one cannot be seen in this surface). However, at the slightly higher energy of $13000 \mathrm{~cm}^{-1}$, above the first SN bifurcation, the structure of the phase space has qualitatively changed and there exists now one extra PO, which is labeled [D]. The SN bifurcation and therefore the birth of 

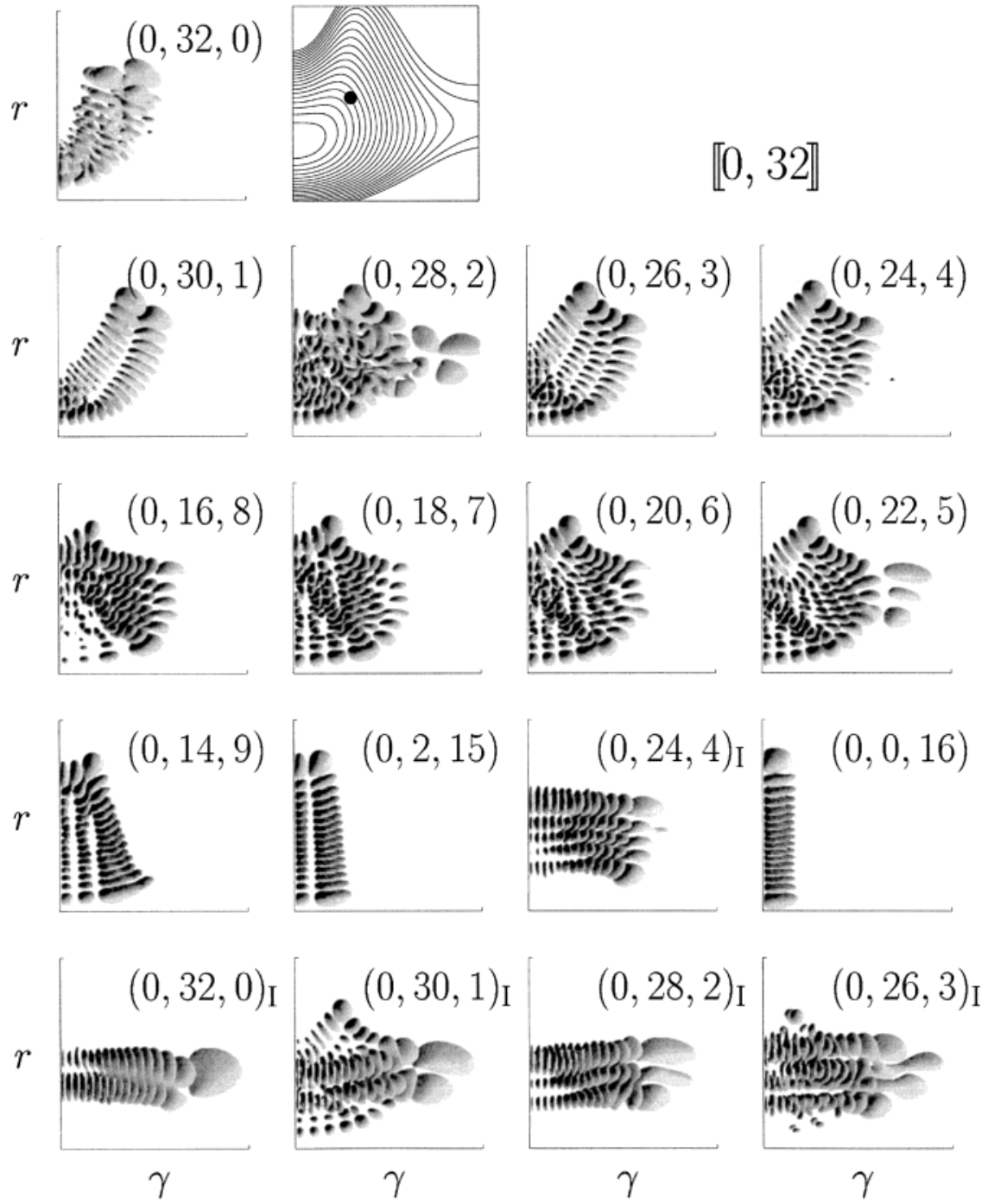

Figure 5. Wave functions for HCP in polyad $[0,32]$. State $(0,32,0)$ is at the top of the polyad and state $(0,32,0)_{\text {I }}$ marks the bottom. For orientation, the potential energy surface is shown in the first row.

the new type of PO occurs at a particular energy. As energy is increased, the stability area around the [D] PO first becomes larger.

At this point, it is necessary to strongly emphasize that, when normal modes and POs begin to deviate, the quantum mechanical dynamics follows the POs. This applies to the principal families of POs as well as to those created at SN bifurcations. Normal modes eventually cease to influence the quantum wave functions, simply because their definition becomes unrealistic with increasing energy. Periodic orbits, on the other hand, continue to be the building blocks of quantum dynamics up to high energies. Within classical mechanics they are exact objects; their calculation does not require any approximation of either the kinetic energy or the potential. Formulated in the language of spectroscopy, this means that a set of quantum numbers assigned to a given state does not indicate the amount of energy put in each normal mode, as it is sometimes understood, but instead indicates the amount of energy traveling along the set of stable POs. In order for assignments to be meaningful, it is not sufficient to just quote the quantum numbers; it is equally important to visualize the POs, which structure the classical phase space.

The "scarring" of quantum mechanical wave functions by stable POs has been amply demonstrated in the literature..$^{70,71,78-80}$ Some examples for HCP are shown in Figure 7. Panels a and c depict, in two different representations, the wave function for state $(0,24,0)$. This state belongs to the progression, which at low excitations is aligned along the reaction coordinate, i.e., the angle $\gamma$. Due to the mixing with the fast mode, $[\mathrm{r}]$ in HCP, it becomes significantly curved at higher energies and avoids the isomerization path. The corresponding PO, termed [B], shows exactly the same behavior. The quantum number $v_{2}$ simply counts the number of nodes along the [B] PO. Panels b and $\mathrm{d}$ depict an example of an isomerization state, i.e., those states that do not exist at low energies and that are born at intermediate energies. It stretches along the isomerization path and so does the PO, called [SN1] here, which belongs to the family that is born at the first saddle-node bifurcation. The same general picture holds for $\mathrm{HOCl}$ and $\mathrm{HOBr}$; examples for $\mathrm{HOCl}$ are given in Figure 11 of ref 23.

Thus, the "new" quantum states born at intermediate energies, which we called isomerization states for HCP and dissociation states for $\mathrm{HOCl} / \mathrm{HOBr}$, are scarred by the saddle-node POs. The birth of the SN trajectories occurs at a specific energy. In quantum mechanics, on the other hand, the new type of wave functions comes into existence in a more gradual manner, as illustrated in Figure 8 of ref 23 for $\mathrm{HOCl}$ and Figure 10 of ref 19 for HCP.

The close correspondence between quantum mechanical states and POs is further illustrated in Figure 8 by showing for HCP 


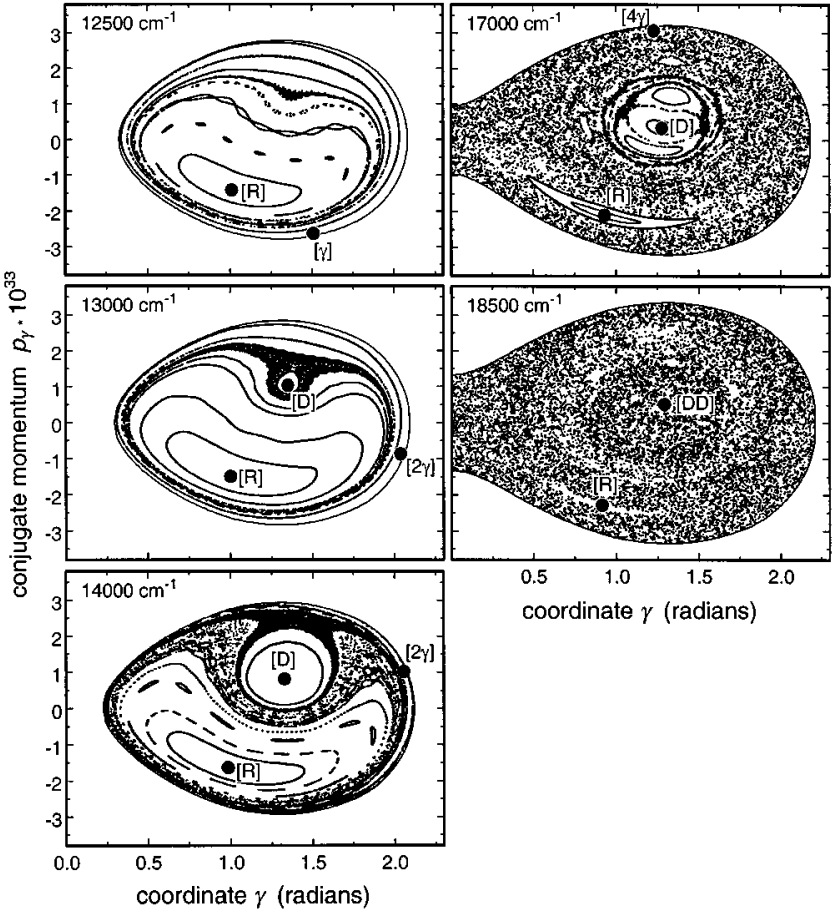

Figure 6. $\left(\gamma, p_{\gamma}\right)$ Poincaré surfaces of sections at different energies for a two-dimensional model of $\mathrm{HOCl}$; the $\mathrm{HO}$ bond distance is fixed at the equilibrium value of the dissociated $\mathrm{OH}$ fragment. The black dots indicate the various stable periodic orbits $(\mathrm{PO})$. $[\mathrm{R}]$ and $[\gamma]$ are the two fundamental POs associated with the slow and the fast degrees of freedom, respectively. [D] is the stable PO born at the first saddlenode (SN) bifurcation, which scars the dissociation states at intermediate energies. [DD] is another stable PO born at a second SN bifurcation, which takes place close to the dissociation threshold. $[2 \gamma]$ and $[4 \gamma]$ are the stable POs obtained from $[\gamma]$ after one and two period doubling bifurcations, respectively; they are not discussed in this article. See ref 63 for more details.

the classical continuation/bifurcation (CB) diagram (lower panel) and its quantum mechanical analogue (upper panel). Plotted are the frequencies of the POs for the slow, [B], and the fast, [r], mode as well as the SN mode, [SN1], [SN2], etc., as functions of energy. Such a CB diagram ${ }^{81,82}$ is quite convenient for getting an overview of the structure of the phase space and the different families of periodic orbits and how they behave as a function of energy. In the quantum mechanical part of the figure, the energy separations between the neighboring states of a given progression are depicted. The isomerization POs and the corresponding quantum states advance along the reaction coordinate, i.e., the coordinate with the largest anharmonicity. As a consequence, the corresponding frequency curves exhibit the largest negative gradient. The large anharmonicity of the isomerization states is the primary cause for the complexity of the energy-level spectrum at intermediate and higher energies in Figure 1; the polyads for $v_{\mathrm{u}}=v_{1}=0$ begin to overlap, and this overlap becomes more and more severe with energy. A plot similar to Figure 8 exists also for $\mathrm{HOCl}^{23}$

In the same manner as the Fermi resonance deflects the slowmode POs and wave functions away from the reaction pathway, higher-order resonances, whose importance increases with energy, might deflect the saddle-node POs and prevent them from further advancing along the reaction coordinate. An illustration thereof is indicated in Figure 7, panel d. This deflection away from the reaction path, however, is again balanced by the appearance of another stable PO named [SN2] at a second saddle-node bifurcation (Figure 8). The [SN2] PO replaces the [SN1] $\mathrm{PO}$ as the backbone for the quantum
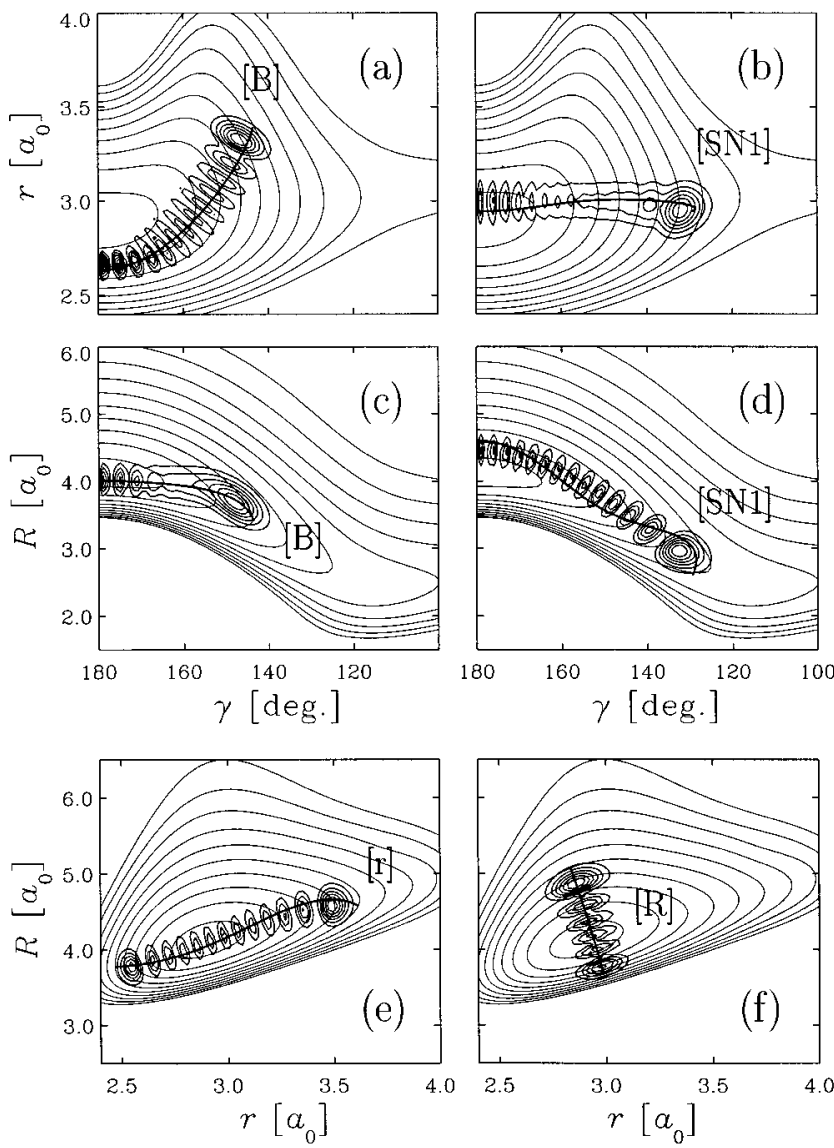

Figure 7. Examples of wave functions and classical periodic orbits for HCP. Reprinted, with permission of Annual Review of Physical Chemistry, from ref 19 (copyright 1999).

isomerization states. The same feature has also been observed in a two-dimensional model of $\mathrm{HOCl}$ (see Figures 1 and 4 of ref 63). One might surmise that, at still higher energies, more and more saddle-node bifurcations occur, which give birth to an increasing number of stable POs, which stretch further and further along the reaction pathway. ${ }^{83}$ However, these successors of the SN POs are very difficult to find, because the classical phase space becomes more and more chaotic with increasing energy.

\section{The Semiclassical Mechanics Point of View: Analysis of an Effective Hamiltonian}

The goal of this section is to demonstrate that (1) effective (or resonance) Hamiltonians, often used by spectroscopists to fit spectra, are able to reproduce the results of exact quantum calculations over very broad energy ranges (including SN bifurcations) and (2) that the semiclassical analysis of these Hamiltonians provides elegant interpretations of even the most subtle features observed in the quantum mechanical spectra in terms of classical quantities.

In contrast to the exact quantum and classical mechanics approaches, which are based on global PESs, the resonance Hamiltonian incorporates severe assumptions regarding the intramolecular energy flow: It assumes that all of the couplings are negligible, except for the Fermi resonance between the fast (f) and the slow (s) modes, whose fundamental frequencies are in near 1:2 degeneracy. For a triatomic molecule with a third, almost uncoupled mode $(\mathrm{u})$, the resonance Hamiltonian in its classical version is written in the form 

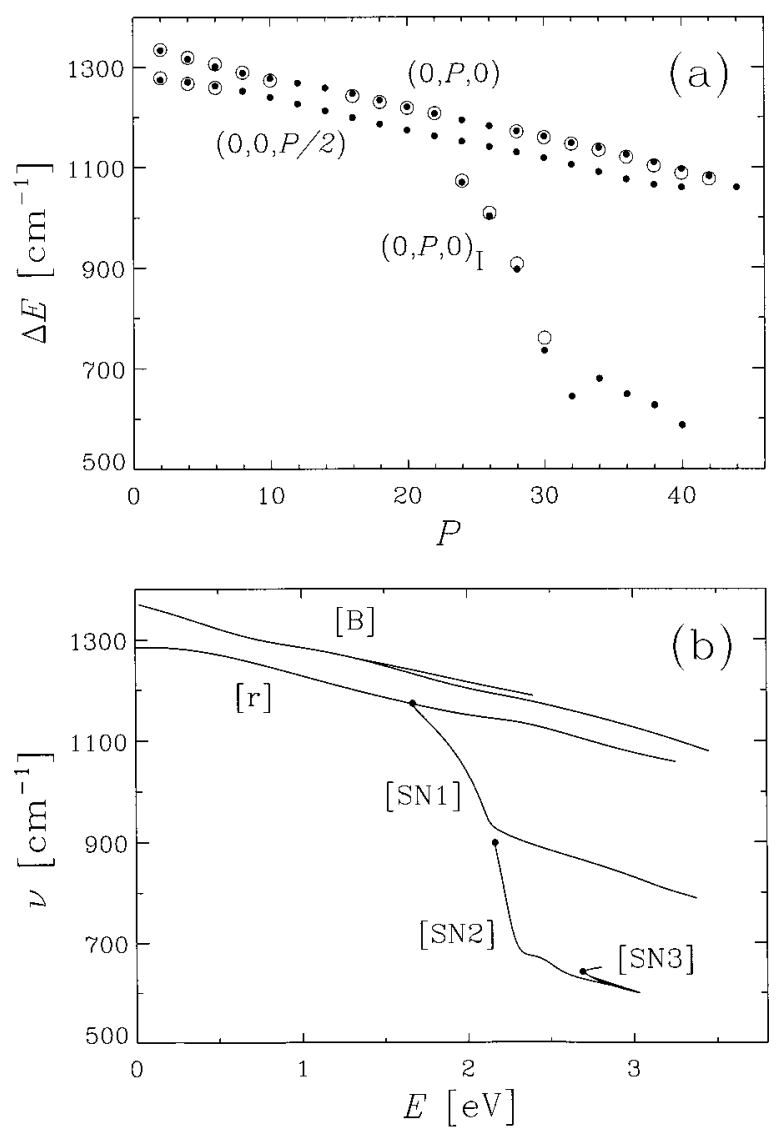

Figure 8. (a) Energy differences between neighboring states of the three progressions $(0, P, 0),(0,0, P / 2)$, and $(0, P, 0)_{\mathrm{I}}$ versus polyad quantum number $P$. The black dots are the quantum mechanical results, whereas the open circles indicate the experimental values. (b) The frequencies of the various families of periodic orbits as functions of the energy. Reprinted, with permission of Annual Review of Physical Chemistry, from ref 19 (copyright 1999).

$$
\begin{gathered}
H=H_{\mathrm{D}}+H_{\mathrm{F}} \\
H_{\mathrm{D}}=\sum_{i=\mathrm{f}, \mathrm{s}, \mathrm{u}} \omega_{i} I_{i}+\sum_{i, j} x_{i j} I_{i} I_{j}+\sum_{i, j, k} y_{i j k} I_{i} I_{j} I_{k}+\ldots \\
H_{\mathrm{F}}=2 I_{\mathrm{f}}^{1 / 2} I_{\mathrm{s}} \cos \left(\varphi_{\mathrm{f}}-2 \varphi_{\mathrm{s}}\right)\left(k+\sum_{i=\mathrm{f}, \mathrm{s}, \mathrm{u}} k_{i} I_{i}+\ldots\right)
\end{gathered}
$$

where the $\left(I_{i}, \varphi_{i}\right)$ are action-angle-like sets of conjugate coordinates built on dimensionless normal coordinates $\left(p_{i}, q_{i}\right)$ according to

$$
\begin{gathered}
I_{i}=\frac{1}{2}\left(q_{i}^{2}+p_{i}^{2}\right) \\
q_{i}=\left(2 I_{i}\right)^{1 / 2} \cos \varphi_{i} \\
p_{i}=-\left(2 I_{i}\right)^{1 / 2} \sin \varphi_{i}
\end{gathered}
$$

$H_{\mathrm{D}}$ is known as the Dunham expansion, while $H_{\mathrm{F}}$ is the Fermi coupling, which describes the flow of energy between the slow and fast degrees of freedom. The spectroscopic constants $\omega_{i}$, $x_{i j}, k, k_{i}$, etc. are obtained by fitting the eigenvalues of exact quantum mechanical calculations (see refs 22, 25, and 41; note that in Table 2 of ref 22 the numerical value for $y_{233}$ should read $0.2503 \mathrm{~cm}^{-1}$ instead of $-0.2503 \mathrm{~cm}^{-1}$ and the parameter $y_{123}=-0.4304 \mathrm{~cm}^{-1}$ has been omitted.). Alternatively, they can be determined by fitting experimental transition energies, if available. The essential point is that the quantum mechanical resonance Hamiltonian is block diagonal in a (direct product) harmonic oscillator basis, each block corresponding to a different polyad $\left[v_{\mathrm{u}}, P\right]$. Stated in other words, $v_{\mathrm{u}}$ and $P$ are exact quantum numbers in the resonance Hamiltonian approach, in contrast to the exact quantum approach, where, because of the nonnegligible coupling between polyads, they are only approximately good quantum numbers.

The resonance Hamiltonian satisfactorily reproduces the exact quantum mechanical energies within a few $\mathrm{cm}^{-1}$ over very broad energy ranges (for example, up to more than $97 \%$ of the dissociation threshold $D_{0}$ for $\mathrm{HOCl}$ ), several hundreds of states, and tens of quanta in the slow mode (see Table 1). ${ }^{22,25,41}$ Even more important is the fact that the wave functions and all their subtleties are also qualitatively reproduced by the Fermi resonance Hamiltonian. Examples for $\mathrm{HOCl}$ are depicted in Figure 9. In particular, the wave functions of the dissociation states and their ordering inside the polyads are correctly described. This is also true for $\mathrm{HOBr}$ and $\mathrm{HCP}$ (see, for example, Figure 1 of ref 41). The good qualitative agreement underlines that the Fermi resonance, the only coupling term in the resonance Hamiltonian, is the principal cause for the saddlenode bifurcations and therefore of the complexity of the quantum mechanical spectrum.

There is, however, one little problem associated with the resonance Hamiltonian approach: Since the resonance Hamiltonian cannot be decomposed into a kinetic energy term and a potential, the relation between the generalized coordinates $\left(q_{\mathrm{u}}\right.$, $\left.q_{\mathrm{s}}, q_{\mathrm{f}}\right)$ and the real coordinates of the molecule, e.g., the Jacobi coordinates $(R, r, \gamma)$ is not unambiguous. Nevertheless, for $\mathrm{HOCl}$, for example, the slow-mode coordinate $q_{\mathrm{s}}$ has predominantly $\mathrm{OCl}$ stretch character. It should not be overlooked that the wave functions from the effective Hamiltonian have, by construction, a symmetry, which is not real. However, these shortcomings do not restrain the usefulness of this approach.

We shall now briefly describe the dynamics of the resonance Hamiltonian, before showing how this knowledge leads to an understanding of the details of the quantum mechanical observations. It is convenient to make a linear transformation from the sets of conjugate coordinates $\left(I_{i}, \varphi_{i}\right)$ defined in eq 3 to new sets $\left(J_{i}, \psi_{i}\right)$, such that

$$
J_{\mathrm{u}}=I_{\mathrm{u}} \quad J_{\mathrm{P}}=2 I_{\mathrm{f}}+I_{\mathrm{s}} \quad J_{0}=2 I_{\mathrm{f}}
$$

for the generalized momenta and

$$
\psi_{\mathrm{u}}=\varphi_{\mathrm{u}} \quad \psi_{\mathrm{P}}=\varphi_{\mathrm{s}} \quad \psi_{0}=\varphi_{\mathrm{s}}-\frac{\varphi_{\mathrm{f}}}{2}
$$

for the generalized coordinates. With these new variables the resonance Hamiltonian of eq 2 is rewritten in the form

$$
\begin{gathered}
H_{\mathrm{D}}=\sum_{i=\mathrm{P}, 0, \mathrm{u}} \Omega_{i} J_{i}+\sum_{i, j} X_{i j} J_{i} J_{j}+\sum_{i, j, k} Y_{i j k} J_{i} J_{j} J_{k}+\ldots \\
H_{\mathrm{F}}=J_{0}^{1 / 2}\left(J_{\mathrm{P}}-J_{0}\right) \cos \left(2 \psi_{0}\right)\left(K+\sum_{i=\mathrm{P}, 0, \mathrm{u}} K_{i} J_{i}+\ldots\right)
\end{gathered}
$$

with trivial linear relations between the spectroscopic coefficients $\omega_{i}, x_{i j}, y_{i j k}, k$, and $k_{i}$ of eq 2 and the coefficients $\Omega_{i}, X_{i j}$, $Y_{i j k}, K, K_{i}$ of eq 6 . Since eq 6 depends neither on $\psi_{\mathrm{u}}$ nor on $\psi_{\mathrm{P}}$, their conjugate momenta $J_{\mathrm{u}}$ and $J_{\mathrm{P}}$ are classical constants of motion, which according to the EBK (Einstein-BrillouinKeller) semiclassical quantization rules ${ }^{84-86}$ can be set to

$$
J_{\mathrm{u}}=v_{\mathrm{u}}+\frac{\eta_{\mathrm{u}}}{2} \quad \text { and } \quad J_{\mathrm{P}}=P+\frac{\eta_{\mathrm{P}}}{2}
$$




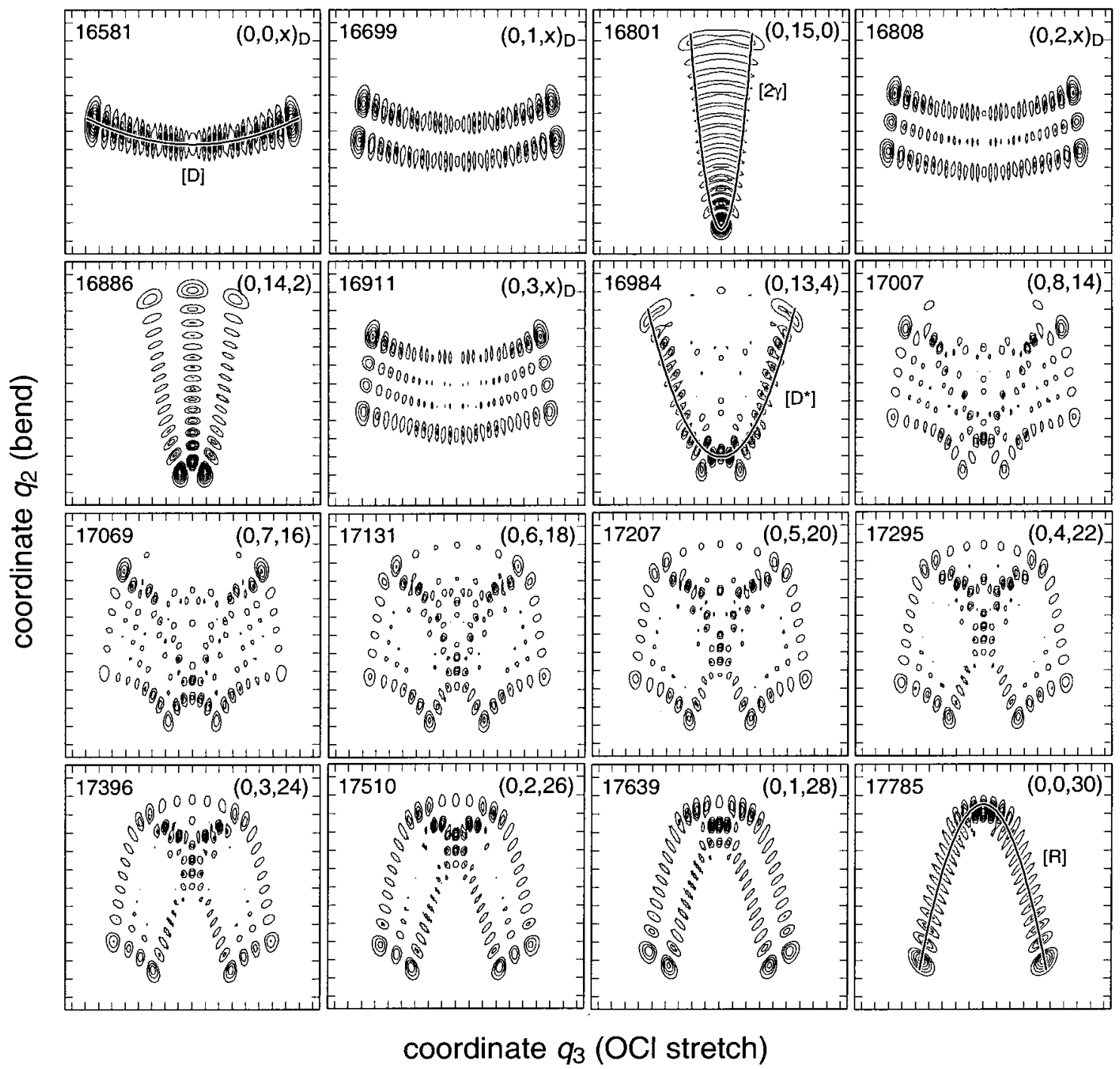

Figure 9. Wave functions for polyad $[0,30]$ of $\mathrm{HOCl}$ as obtained from the effective Hamiltonian. The coordinate $q_{3}$ ranges from -9.5 to +9.5 , and $q_{2}$ ranges from -6.7 to +6.7 . Note that the underlying Hamiltonian was fitted to the quantum mechanical calculations of Peterson et. al., ${ }^{21}$ who used a slightly different potential energy surface than the one employed by Weiss et al. ${ }^{23}$ Therefore, the order of the states at high energies slightly differs from the order in Figure 4. The continuous lines in four of the panels indicate the corresponding periodic orbits (see Figure 10).

respectively, with $v_{\mathrm{u}}$ and $P$ being positive integers. $\eta_{\mathrm{u}}$ and $\eta_{\mathrm{P}}$ depend on the degree of degeneracy of the three normal modes: $\eta_{\mathrm{u}}$ is equal to 1 for the three molecules $\mathrm{HCP}, \mathrm{HOCl}$, and $\mathrm{HOBr}$, while $\eta_{\mathrm{P}}$ is equal to 4 for $\mathrm{HCP}$ and to 3 for $\mathrm{HOCl}$ and $\mathrm{HOBr}$. The only nontrivial equations of motion are those concerning $\left(J_{0}, \psi_{0}\right)$. The corresponding quantization condition reads

$$
\mathscr{J}=\frac{1}{2 \pi} \int_{0 \leq \psi_{0} \leq \pi} J_{0} \mathrm{~d} \psi_{0}=n_{0}+\frac{1}{2}
$$

where $n_{0}$ can be any positive or negative integer number. Stated in other words, the classical resonance Hamiltonian in eq 6 can be considered as an effective one-dimensional problem in the variables $\left(J_{0}, \psi_{0}\right)$, with coefficients depending parametrically, through eq 7 , on the particular polyad.

The cornerstones of the subsequent analysis are the fixed points of this Hamiltonian, that is, according to the Hamilton's equations of motion, those points which satisfy

$$
\frac{\partial H}{\partial J_{0}}=\frac{\partial H}{\partial \psi_{0}}=0
$$

The fixed points in the $\left(J_{0}, \psi_{0}\right)$ space define POs in the space of the dimensionless coordinates, which are coupled by the Fermi resonance, that is $\left(p_{\mathrm{f}}, p_{\mathrm{s}}, q_{\mathrm{f}}, q_{\mathrm{s}}\right)$. As long as the third mode, $\mathrm{u}$, remains essentially decoupled from the modes involved in the Fermi resonance, these POs with reduced dimensionality are sufficient for understanding the properties of the quantum wave functions. In comparison with the six-dimensional POs obtained from the exact classical mechanics study using the full PES, the semiclassical POs are determined for a particular pair of quantum numbers $v_{\mathrm{u}}$ and $P$, i.e., for a particular polyad $\left[v_{\mathrm{u}}\right.$, $P]$, instead of just the energy. Examples for the polyad $\left[v_{\mathrm{u}}, P\right]$ $=[0,30]$ of $\mathrm{HOCl}$ are plotted in Figure 9 on top of the wave functions obtained from the effective Hamiltonian.

Why are the isomerization and dissociation states found at the low-energy ends of the polyads? Plotting, for given values of $v_{\mathrm{u}}$, the energies of the POs as a function of the polyad number $P$, which in principle can be any real number, provides an immediate answer (Figure 10). The energies of the quantum states for a particular $P$ (30 for $\mathrm{HOCl}$ in the figure), aligned on a vertical line, are always confined to the region between the two stable POs with the lowest and the highest energy. For low values of $P$, there exist only two stable POs, which are 
(a) $\mathrm{HCP}$

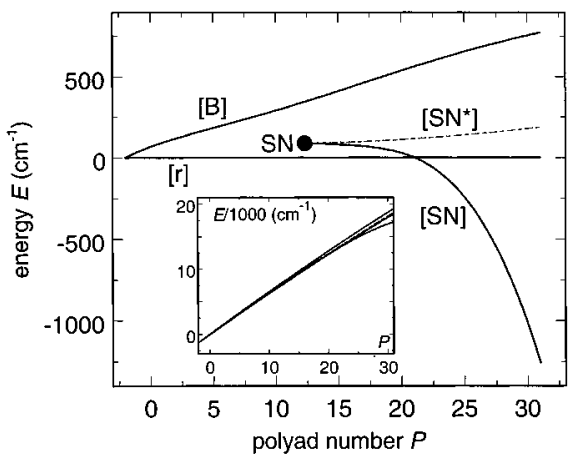

(b) $\mathrm{HOCl}$

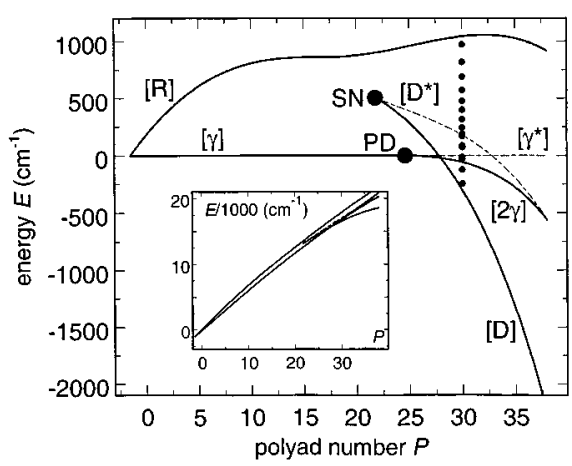

Figure 10. Energies of the periodic orbits (PO) as functions of the polyad number $P$ with respect to the energy of the PO corresponding to the fast mode ([r] for HCP and $[\gamma]$ for $\mathrm{HOCl}$ ). The insets show the absolute energies. The heavy dots marked SN indicate the saddle-node bifurcations, and $\mathrm{PD}$ for $\mathrm{HOCl}$ marks a period doubling bifurcation. Stable periodic orbits are indicated by the solid lines, while unstable periodic orbits created at the saddle-node bifurcation or emerging from the period doubling bifurcation are indicated by the dashed curves and by the asterisks, e.g., [D*]. The vertically arranged dots for $\mathrm{HOCl}$ and $P=30$ indicate the quantum mechanical energy levels. In both cases, $v_{\mathrm{u}}=v_{1}=0$.

associated with the slow (highest energy) and fast (lowest energy) modes, respectively. However, above the first SN bifurcation (the corresponding values of $P$ are listed in Table 1) there exists a third stable PO, which scars the isomerization/ dissociation states and is named, according to the classical mechanics calculations, [SN] for $\mathrm{HCP}$ and [D] for $\mathrm{HOCl}$ and $\mathrm{HOBr}$. Although the stable PO appearing at the saddle-node bifurcation is born inside the classically accessible range, because of the large anharmonicity its energy quickly drops below the energy of the PO, which is associated with the fast degree of freedom. The same development occurs in the quantum mechanical spectrum, i.e., the isomerization/ dissociation states, which are also born inside the polyad, rapidly move to the lower end of the polyad (a clear illustration thereof is provided in Figure 10 of ref 19). The net result is that states stretching mainly along the reaction pathway, which due to the mixing with the fast mode have disappeared from the top of the polyads, reappear at the bottom as a consequence of the $\mathrm{SN}$ bifurcations. Although this might seem, from the quantum mechanical point of view, like a simple crossing due to the very different anharmonicities, the classical and semiclassical pictures illustrate that, because of the Fermi resonance, this is a quite involved process; it requires, first, a saddle-node bifurcation to take place and, second, that the stable PO born at this bifurcation advances along the reaction pathway and thus becomes the lowenergy boundary of the classically accessible phase space.
There are further essential observations explained by the semiclassical approach. For example, how can one understand that the increase of the number of SN states is counterbalanced by a decrease of the number of states belonging to the normal progression? A plot of the action integral $\mathscr{L}$ as function of energy $E$ as well as polyad number $P$ provides an answer. In Figure 11 we depict for $\mathrm{HOCl}$ a sequence of $\not{X} E)$ for four different values of $P ; v_{\mathrm{u}}=0$ in all cases. The action integral for the lowest polyad, located below the value of $P$ where the saddlenode bifurcation occurs, $P_{\mathrm{SN}}$, consists of a single branch, which extends between the two stable POs found at this energy. According to the semiclassical quantization rule in eq 8 , the quantum states correspond to half-integer values of $\mathscr{J}$ and are indicated by the black dots. This single branch supports the states belonging to the normal progressions, which are found below $P_{\mathrm{SN}}$.

As $P$ increases and approaches $P_{\mathrm{SN}}$ from below, a point with increasingly larger derivative develops in the plot of $\mathscr{X} E$ ) (see $P=21$ in Figure 11). Just at the bifurcation, two developments are observed. First, the derivative becomes infinite and the single branch splits into two, which further and further separate as $P$ increases. This discontinuity arises at the energy of the unstable PO (labeled by $\left[\mathrm{D}^{*}\right]$ ), which is born at the bifurcation simultaneously with the new stable PO (labeled by [D]). Most importantly, these two branches still support only states belonging to the normal progression, and the semiclassical quantization rule in eq 8 applies to each branch separately. As the gap in $\mathscr{J}$ between the two branches becomes larger, it encompasses an increasing number of half-integer values, with the result that more and more members of the normal progression disappear, simply because eq 8 has no real solution for these values of $n_{0}$.

The second phenomenon, which takes place at the bifurcation, is the appearance of a new branch with positive values of $\mathscr{X} E)$, which extends between the energies of the two POs born at the bifurcation, the stable PO and the unstable one (see $P=24$ in Figure 11). In contrast to the two other branches, this one supports the members of the new progression of dissociation states, indicated by the open diamonds. As $P$ increases, this branch extends further and further and the semiclassical quantization rule ensures that a member of the new progression is created each time the new branch encompasses an additional half-integer value of $\mathscr{W}$. At last, it should be noted that there exists a given energy range where the two branches coexist at the same energy. This energy range extends from the unstable PO born at the bifurcation down to either the stable PO born at the bifurcation or the stable PO associated with the fast degree of freedom, depending on which one is higher in energy. Since one branch supports members of the normal progression and the other branch members of the new progression, the two progressions overlap at the bottom of the polyad. There are even finer details revealed by the semiclassical approach, which are also seen in the exact quantum wave functions; they are partly caused by the occurrence of a period doubling bifurcation slightly above the $\mathrm{SN}$ bifurcation (Figure 10 and $P=30$ in Figure 11). However, because of lack of space the reader is referred to the original literature. ${ }^{22,41}$

\section{Some Notes on Vibrational Chaos}

The purpose of this section is to discuss briefly the question of vibrational chaos and particularly to emphasize that for highly excited small molecules classical and quantum mechanics do not necessarily agree when describing a system as "regular" or "chaotic". A more thorough discussion of chaos, concerning $\mathrm{HOCl}$, can be found in ref 63 and the references therein. 


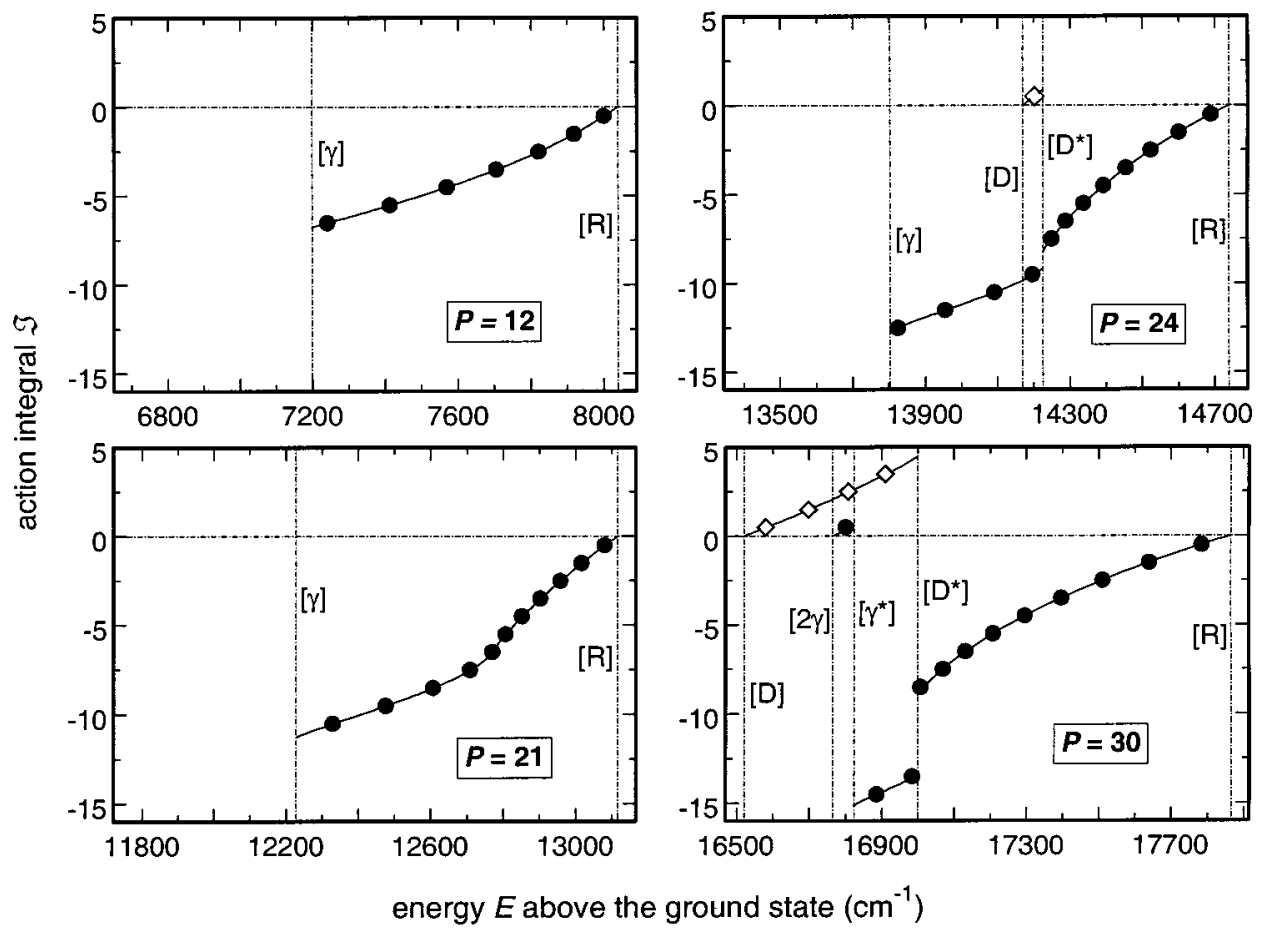

Figure 11. Action integral $\mathscr{Z}(E)$ for $\mathrm{HOCl}$ and four polyads. The vertical lines indicate the energies of the various periodic orbits. The quantum mechanical states belonging to the normal and to the new progressions are indicated by the filled circles and the open diamonds, respectively.

Chaos in classical mechanics is well-defined: A region of the phase space is chaotic if two neighboring trajectories launched in this region separate exponentially. There exist several reliable methods for checking and visualizing chaos, for example, the computation of Lyapunov exponents and the plot of Poincaré surfaces of section. The notion of "quantum chaos" is less well-defined. It relies on the proposition made by Percival ${ }^{87,88}$ that the bound-state energy spectrum might be divided into two parts, a regular and an irregular one, which would correspond, respectively, to classical regimes of integrable and chaotic motions. The notion of an irregular (or chaotic) spectrum indicates that the underlying classical chaotic motion has a hallmark in the quantum mechanical properties of the system.

It has been suggested that the distributions of the level spacings, like the nearest-neighbor level spacing or the spectral rigidity ${ }^{3,69,89,90}$ for example, distinguishes regular and irregular spectra. These tests are, however, of little help for the systems studied in this article because of three reasons. First, the number of states is still too small, so that most distributions are obscured by noise. Second, all the tests rely on the assumption that the density of states remains nearly constant in the investigated energy window, whereas this is not the case when an isomerization saddle or a dissociation limit is approached. As a consequence, the results depend sensitively on the particular procedure for unfolding the spectra. However, even if one would find numerical tricks for solving these problems, a major objection would still hold: The degree of (quantum) chaos of a system can be estimated by how its spectrum evolves from a Poisson to a Wigner distribution as energy increases. The spectrum of a triatomic molecule with a hydrogen atom (and consequently one fundamental frequency much larger and fairly decoupled from the two other ones) is, however, neither Poisson at low energies nor Wigner at high energies, so that no firm conclusions can be drawn from the examination of level-spacing distributions. Thus, statistical arguments fail to give a clear-cut indication regarding the (ir)regularity of a spectrum, especially for the systems discussed in this overview.
Alternatively, one can examine the nodal lines of quantum wave functions. Indeed, when increasing the energy, one usually observes a change in the nodal structure, from a regular pattern of intersecting nodal lines to an irregular structure with apparently no crossing of nodal lines. However, Pechukas ${ }^{91}$ has shown that one cannot decide on the basis of this observation alone, whether the change is due to the underlying chaotic classical dynamics or due to an increase of the nonseparability of the system, which is not at all related to chaos. On the other hand, the opposite observation that most wave functions have a sufficiently clear nodal pattern should be considered as an indication for the underlying classical dynamics to be principally regular.

Let us test this idea by a two-dimensional model for $\mathrm{HOCl}$ in which the $\mathrm{HO}$ bond length is frozen. As discussed in ref 63, the quantum spectrum and wave functions closely resemble those of the full three-dimensional calculation for $v_{\mathrm{u}}=v_{1}=0$. The structure of the classical phase space for the $2 \mathrm{D}$ model is illustrated by Poincaré surfaces of section (SOS's) displayed in Figure 6 for several energies. If the dynamics is regular, the motion is confined to a torus and the trajectory cuts the section in one or several closed curves. In contrast, areas filled with randomly distributed points indicate that tori no longer exist in these regions and that the dynamics is chaotic. Examination of the SOS's at $E=13000$ and $14000 \mathrm{~cm}^{-1}$ shows that the saddlenode bifurcation, where the dissociation PO ([D]) is born, plays a fundamental role in the onset of chaotic motion; the first extended region filled with chaotic trajectories clearly develops around the unstable PO created at this bifurcation together with the stable PO [D]. At $E=17000 \mathrm{~cm}^{-1}$, the largest part of the phase space is already chaotic and at $E=18500 \mathrm{~cm}^{-1}$, which is about $800 \mathrm{~cm}^{-1}$ below the dissociation threshold, all of the regular regions have disappeared, except for a very thin crescent around $[R]$ and an even smaller, almost indistinguishable island around [DD]. Also this last pocket of regularity eventually vanishes, around $18800 \mathrm{~cm}^{-1}$, and the SOS's henceforth look totally chaotic. 
The finding that close to the dissociation threshold the entire classical phase space is chaotic, which is intuitively expected, is in contrast with three observations made in the quantum mechanical analysis of the 2D model. First, the nodal lines of nearly all the wave functions up to the dissociation threshold are sufficiently regular to allow an unambiguous assignment. Second, the quantal SOS's based on Husimi distribution functions are much more regular than the classical ones (see Figures 6 and 7 of ref 63). The Husimi functions are constructed with the help of the exact quantum wave functions. Last, but not least, the ability to reproduce the ab initio quantum energies and wave functions with a fully integrable Hamiltonian up to $400 \mathrm{~cm}^{-1}$ below the dissociation threshold with an error of only a few $\mathrm{cm}^{-1}$ clearly suggests that, from the quantum point of view, the nonintegrable part of the Hamiltonian is merely a small perturbation around a basically integrable system, even in the case where classical chaos is fully developed and occupies all the available phase space (see also ref 92). The same discussion and conclusions also hold for $\mathrm{HCP}$ and $\mathrm{HOBr}$ (see Table 1) and $\mathrm{HCN} / \mathrm{CNH}^{31}{ }^{31}$ From the mathematical point of view, this disagreement between classical dynamics (chaos) and quantum dynamics (regularity) indicates that these triatomic molecules are not "semiclassical" or, in other words, that the system still does not approach the mathematical limit $\hbar \rightarrow 0$, despite the fact that nearly forty quanta of energy are deposited in the reactive mode.

\section{Discussion}

That changes in the classical phase space structure may leave a clear fingerprint in the corresponding quantum mechanical spectrum is certainly not new. Many previous studies have indeed focused on the changes in spectra induced by the normalto-local bifurcation. 2,37,38,93-106 As emphasized earlier in this article, the condition for a bifurcation to take place is that at least two modes are coupled by a systematic nonlinear offdiagonal resonance. It must be emphasized that an accidental near degeneracy of some pairs of states, which is absolutely unavoidable, does not lead to systematic changes of the spectrum over wide regions of energy (see, e.g., HCO and refs 27 and 107).

The most frequently encountered couplings are the 1:1 Darling-Dennison ${ }^{108}$ and the 1:2 Fermi ${ }^{54,55}$ resonances. The dynamics induced by these two generic resonances has been studied in detail by Kellman and co-workers in a long series of articles, $, 7,8,109-113$ where the connection with mathematical tools such as the catastrophe map and the theory of elementary bifurcations is nicely discussed and illustrated, and more recently by Joyeux. ${ }^{114-117}$ A nonexhaustive list of the molecules for which the 1:1 Darling-Dennison coupling plays a fundamental role includes water, ${ }^{66}$ ozone, ${ }^{67}$ the $\mathrm{CH}$ stretches of acetylene, ${ }^{7}$ hydrogen sulfide, ${ }^{68} \mathrm{DCO},{ }^{28,29}$ and $\mathrm{SO}_{2} .{ }^{118}$ While the dynamics of DCO is governed by a SN bifurcation, ${ }^{107}$ all of the other molecules experience a normal-to-local bifurcation at low energies.

Prior to our analysis of phosphaethyne (HCP), hypochlorous acid $(\mathrm{HOCl})$, and hypobromous acid $(\mathrm{HOBr})$, the Fermi resonance was shown to have a fundamental influence on the spectra of carbon dioxide,,${ }^{9,10,119-121}$ carbone disulfide, ${ }^{122-125}$ and the $\mathrm{CH}$ chromophore in substituted methanes $\left(\mathrm{HCX}_{3}\right.$, for a review see ref 126 and references therein). The dynamics of $\mathrm{CO}_{2}, \mathrm{CS}_{2}$, and the substituted methanes is dominated by perioddoubling bifurcations, ${ }^{111}$ although systems with a Fermi resonance can undergo period-doubling as well as SN bifurcations. As shown in ref 116, the reason for this is that a strong Fermi resonance leads to a period-doubling bifurcation at low energies, whereas a weaker resonance leads to a $\mathrm{SN}$ bifurcation only at higher energies. Since the older studies ${ }^{7,8,109-117}$ were based on model Hamiltonians fitted to a relatively small number of experimentally observed transitions, it is not surprising that systems with a strong, and therefore easy to detect, resonance were focused on. Our ability to clearly identify $\mathrm{SN}$ bifurcations in the vibrational dynamics of $\mathrm{HCP}, \mathrm{HOCl}$, and $\mathrm{HOBr}$, for which the Fermi resonance is much weaker than for $\mathrm{CO}_{2}, \mathrm{CS}_{2}$, and the substituted methanes, relies on the fact that we were able to compute and investigate very large sets of quantum states, up to extremely high energies.

The only molecule up to now, for which an SN bifurcation has been unambiguously detected, is HCP. ${ }^{19,40}$ Another molecule, which shows a SN bifurcation ${ }^{107}$ and for which a large number of states have been measured ${ }^{28}$ as well as calculated, ${ }^{29}$ is DCO. The progression born at the $\mathrm{SN}$ bifurcation is the DC stretch mode, i.e., the dissociation mode. Since the dissociation energy is unusually small, this progression is very short and therefore the effect of the SN bifurcation on the spectrum is not pronounced.

In this article we only discussed the spectra with no excitation in the uncoupled mode, i.e., the $\mathrm{CP}$ stretch in $\mathrm{HCP}$ and the $\mathrm{HO}$ stretch in $\mathrm{HOCl}$ and $\mathrm{HOBr}$. Although these modes are only weakly coupled to the Fermi resonance modes, they do have a noticeable influence on the spectra and the appearance of the SN states. For more details see refs 41 and 65 for HCP and for $\mathrm{HOCl}$.

There are a few recent studies that generalize the results presented here. First of all, some systems, like water, ${ }^{127,128}$ the bending vibrations of acetylene, ${ }^{129,130}$ or $\mathrm{DCO},{ }^{131}$ require that at least two resonances be taken into account for a correct description. Although somewhat more tedious than for molecules with a single prominent resonance, the dynamical investigation of such systems is still possible, as shown by Sibert and McCoy ${ }^{132}$ and Jacobson et al. ${ }^{133}$ for the bending vibrations of acetylene and Jung et al. ${ }^{134}$ for $\mathrm{CHBrClF}$. Moreover, the description of the reactive mode in terms of harmonic oscillators becomes insufficient for energies substantially higher than those discussed here. Jacobson and Child ${ }^{135,136}$ have solved this difficulty in the case of HCP by describing the bending motion as an internal rotor. They have shown that the investigation of the quantum and classical dynamics of the interaction between a hindered internal rotor mode and a stretch mode provides additional and original information on the bond-breaking internal rotation of $\mathrm{HCP}$.

At last, it is interesting to note that $\mathrm{SN}$ bifurcations are not only observed in systems with few degrees of freedom but also in infinite one-dimensional systems. Sievers and Takeno ${ }^{137}$ originally noticed and MacKay and Aubry ${ }^{138}$ and Aubry ${ }^{139}$ proved that spatially localized motion periodic in time, called discrete breathers, exists in infinite periodic lattices with nonlinear terms and with conditions close to the decoupled limit. These results have been recently extended to one-dimensional chains of random anharmonic oscillators by Kopidakis and Aubry. ${ }^{140,141}$ They have constructed CB diagrams and showed that the discrete breathers are associated with POs originating from SN bifurcations.

Acknowledgment. R.S. acknowledges financial support by the Deutsche Forschungsgemeinschaft. We are grateful to the various students and postdocs involved in these studies over a number of years, in particular H.-M. Keller, C. Beck, J. Weiss, J. Hauschildt, S. Stamatiadis, and T. Azzam. Many stimulating 
discussions with colleagues such as Profs. Field, Ishikawa, and Koput are also greatly acknowledged.

\section{References and Notes}

(1) Wilson, E. B.; Decius, J. C.; Cross, P. C. Molecular Vibrations; McGraw-Hill: New York, 1954.

(2) Child, M. S. Semiclassical Mechanics with Molecular Applications; Clarendon Press: Oxford, U.K., 1991.

(3) Tabor, M. Chaos and Integrability in Nonlinear Dynamics; Wiley: New York, 1989. 991.

(4) Haake, F. Quantum Signatures of Chaos; Springer: New York,

(5) Henon, M.; Heiles, C. Astron. J. 1964, 69, 73

(6) Noid, D. W.; Marcus, R. A. J. Chem. Phys. 1977, 67, 559

(7) Kellman, M. E. J. Chem. Phys. 1985, 83, 3843.

(8) Kellman, M. E.; Lynch, E. D. J. Chem. Phys. 1986, 85, 7216.

(9) Joyeux, M. Chem. Phys. 1997, 221, 269.

(10) Joyeux, M. Chem. Phys. 1997, 221, 287.

(11) Farantos, S. C. Laser Chem. 1993, 13, 87.

(12) Yarkony, D. R., Ed.; Modern Electronic Structure Theory; World Scientific: Singapore, 1995; Parts I and II.

(13) Siebert, R.; Schinke, R.; Bittererová, M. Phys. Chem. Chem. Phys. 2001, 3, 1795 .

(14) Bačić, Z.; Light, J. C. Annu. Rev. Phys. Chem. 1989, 40, 469.

(15) Tennyson, J.; Miller, S.; Henderson, J. R. In Methods in Compu-

tational Chemistry: Molecular Vibrations; Wilson, S., Ed.; Plenum: New York, 1992; Vol. 4, p 91.

(16) Carrington, T., Jr. In Encyclopedia of Computational Chemistry; Schleyer, P., Allinger, N. L., Clark, T., Gasteiger, J., Kollman, P. A., Schaefer, H. F., III, Schreiner, P. R., Eds.; John Wiley \& Sons: Chichester, U.K., 1998; Vol. 5, p 3157.

(17) Davis, M. J. Int. Rev. Phys. Chem. 1995, 14, 15

(18) Beck, C.; Schinke, R.; Koput, J. J. Chem. Phys. 2000, 112, 8446.

(19) Ishikawa, H.; Field, R. W.; Farantos, S. C.; Joyeux, M.; Koput, J.;

Beck, C.; Schinke, R. Annu. Rev. Phys. Chem. 1999, 50, 443.

(20) Bredenbeck, J.; Beck, C.; Schinke, R.; Stamatiadis, S.; Farantos,

S. C.; Joyeux, M. J. Chem. Phys. 2000, 112, 8855.

(21) Peterson, K. A.; Skokov, S.; Bowman, J. M. J. Chem. Phys. 1999 111,7446

(22) Jost, R.; Joyeux, M.; Skokov, S.; Bowman, J. M. J. Chem. Phys. $1999,111,6807$.

(23) Weiss, J.; Hauschildt, J.; Grebenshchikov, S. Y.; Düren, R.; Schinke, R.; Koput, J.; Stamatiadis, S.; Farantos, S. C. J. Chem. Phys. 2000, 112, 77 .

(24) Peterson, K. A. J. Chem. Phys. 2000, 113, 4598.

(25) R. Schinke and co-workers, work in progress.

(26) Werner, H.-J.; Bauer, C.; Rosmus, P.; Keller, H.-M.; Stumpf, M.; Schinke, R. J. Chem. Phys. 1995, 102, 3593.

(27) Keller, H.-M.; Flöthmann, H.; Dobbyn, A. J.; Schinke, R.; Werner, H.-J.; Bauer, C.; Rosmus, P. J. Chem. Phys. 1996, 105, 4983.

(28) Stöck, C.; Li, X.; Keller, H.-M.; Schinke, R.; Temps, F. J. Chem. Phys. 1997, 106, 5333 .

(29) Keller, H.-M.; Schröder, T.; Stumpf, M.; Stöck, C.; Temps, F.; Schinke, R.; Werner, H.-J.; Bauer, C.; Rosmus, P. J. Chem. Phys. 1997, 106,5359 .

(30) Bowman, J. M.; Gazdy, B.; Bentley, J. A.; Lee, T. J.; Dateo, C. E. J. Chem. Phys. 1993, 99, 308. 7165.

(31) Sugny, D.; Joyeux, M.; Sibert, E. L. J. Chem. Phys. 2000, 113,

(32) Golubistsky, M.; G.Schaeffer, D. Singularities and Groups in Bifurcation Theory; Springer: New York, 1985; Vol. I.

(33) Berge, P.; Pomeau, Y.; Vidal, C. Order within Chaos; Wiley: New York, 1984.

(34) Ishikawa, H.; Chen, Y.-T.; Ohshima, Y.; Wang, J.; Field, R. W. J. Chem. Phys. 1996, 105, 7383.

(35) Dai, H.-L., Field, R. W., Eds.; Molecular Dynamics and Spectroscopy by Stimulated Emission Pumping; World Scientific: Singapore, 1995.

(36) Ishikawa, H.; Nagao, C.; Mikami, N.; Field, R. W. J. Chem. Phys. $1998,109,492$

(37) Child, M. S.; Halonen, L. Adv. Chem. Phys. 1984, 57, 1.

(38) Child, M. S. Acc. Chem. Res. 1985, 18, 45.

(39) Halonen, L. Adv. Chem. Phys. 1998, 104, 41.

(40) Ishikawa, H.; Nagao, C.; Mikami, N.; Field, R. W. J. Chem. Phys.

1997, 106, 2980.

(41) Joyeux, M.; Sugny, D.; Tyng, V.; Kellman, M. E.; Ishikawa, H.;

Field, R. W.; Beck, C.; Schinke, R. J. Chem. Phys. 2000, 112, 4162.

(42) Abel, B.; Charvát, A.; Deppe, S. F.; Hamann, H. H. Ber. BunsenGes. Phys. Chem. 1997, 101, 329.

(43) Hamann, H. H.; Charvát, A.; Abel, B.; Kovalenko, S. A.; Kachanov, A. A. J. Chem. Phys. 1997, 106, 3103. 976.
(45) Callegari, A.; Rebstein, J.; Jost, R.; Rizzo, T. R. J. Chem. Phys. 1999, 111, 7359

(46) Weiss, J.; Hauschildt, J.; Schinke, R.; Haan, O.; Skokov, S. Bowman, J. M.; Mandelshtam, V. A.; Peterson, K. A. To be published. (47) Hollebeek, T.; Ho, T.-S.; Rabitz, H. Annu. Rev. Phys. Chem. 1999, 50,537 .

(48) Schatz, G. C. In Reaction and Molecular Dynamics; Lagana, A., Riganelli, A., Eds.; Springer: Berlin, 2000.

(49) Lehmann, K. K.; Ross, S. C.; Lohr, L. L. J. Chem. Phys. 1985, 82, 4460.

(50) Lee, T. J. J. Phys. Chem. 1994, 98, 3697.

(51) Hernandez, M.; Redondo, C.; Lagana, A.; Aspuru, G.; Rosi, M.; Sgamellotti, A. J. Chem. Phys. 1996, 105, 2710.

(52) Füsti-Molnar, L.; Szalay, P. G.; Balint-Kurti, G. G. J. Chem. Phys. $1999,110,8448$.

(53) Lee, T. J.; Parthiban, S.; Head-Gordon, M. Spectrochim. Acta A 1999, 55,561

(54) Fermi, E. Z. Phys. 1931, 71, 250.

(55) Dennison, D. M. Phys. Rev. 1932, 41, 304

(56) Carter, S.; Handy, N. C. Comput. Phys. Rep. 1986, 5, 117.

(57) Light, J. C. In Time-Dependent Quantum Molecular Dynamics;

Broeckhove, J., Lathouwers, L., Eds.; Plenum Press: New York, 1992.

(58) Wall, M. R.; Neuhauser, D. J. Chem. Phys. 1995, 102, 8011.

(59) Mandelshtam, V. A.; Grozdanov, T. P.; Taylor, H. S. J. Chem. Phys. 1995, 103, 10074.

(60) Dobbyn, A. J.; Stumpf, M.; Keller, H.-M.; Schinke, R. J. Chem. Phys. 1995, 103, 9947.

(61) Schinke, R.; Beck, C.; Grebenshchikov, S. Y.; Keller, H.-M. Ber Bunsen-Ges. Phys. Chem. 1998, 102, 593.

(62) Baer, T.; Hase, W. L. Unimolecular Reaction Dynamics; Oxford University Press: Oxford, U.K., 1996.

(63) Joyeux, M.; Sugny, D.; Lombardi, M.; Jost, R.; Schinke, R.; Skokov, S.; Bowman, J. M. J. Chem. Phys. 2000, 113, 9610.

(64) Farantos, S. C.; Keller, H.-M.; Schinke, R.; Yamashita, K.; Morokuma, K. J. Chem. Phys. 1996, 104, 10055.

(65) Beck, C.; Keller, H.-M.; Grebenshchikov, S. Y.; Schinke, R.; Farantos, S. C.; Yamashita, K.; Morokuma, K. J. Chem. Phys. 1997, 107, 9818.

(66) Baggott, J. E. Mol. Phys. 1988, 65, 739.

(67) Lehmann, K. K. J. Phys. Chem. 1984, 88, 1047.

(68) Bykov, A. D.; Naumenko, O. V.; Smirnov, M. A.; Sinitsa, L. N.;

Brown, L. R.; Crisp, J.; Crisp, D. Can. J. Phys. 1994, 72, 989.

(69) Gutzwiller, M. C. Chaos in Classical and Quantum Mechanics; Springer: New York, 1990

(70) Gomez Llorente, J. M.; Pollak, E. Annu. Rev. Phys. Chem. 1992, $43,91$.

(71) Taylor, H. S. In Molecular Dynamics and Spectroscopy by Stimulated Emission Pumping; Dai, H., Field, R. W., Eds.; World Scientific: Singapore, 1995.

(72) Guckenheimer, J.; Holmes, P. Nonlinear Oscillations, Dynamical Systems, and Bifurcations of Vector Fields; Springer-Verlag: Berlin, 1983.

(73) Farantos, S. C. Int. Rev. Phys. Chem. 1996, 15, 345.

(74) Farantos, S. C. Comput. Phys. Comm. 1998, 108, 240.

(75) Vrahatis, M. N.; Perdiou, A. E.; Kalantonis, V. S.; Perdios, E. A.; Papadakis, K.; Prosmiti, R.; Farantos, S. C. Comput. Phys. Commun. 2001 138,53 .

(76) Weinstein, A. Inv. Math. 1973, 20, 47.

(77) Moser, J. Commun. Pure Appl. Math 1976, 29, 727.

(78) Heller, E. J.; Tomsovic, S. Phys. Today 1993, 46 (7), 38

(79) Deleon, N.; Davis, M. J.; Heller, E. J. J. Chem. Phys. 1984, 80, 794.

(80) Founargiotakis, M.; Farantos, S. C.; Contopoulos, G.; Polymilis, C. J. Chem. Phys. 1989, 91, 1389 .

(81) Wiggins, S. Global Bifurcations and Chaos; Springer-Verlag: Berlin, 1988.

(82) Wiggins, S. Introduction to Applied Nonlinear Dynamical Systems and Chaos; Springer: New York, 1990.

(83) Founargiotakis, M.; Farantos, S. C.; Skokos, M. S.; Contopoulos,

G. Chem. Phys. Lett. 1997, 277, 456.

(84) Einstein, A. Verh. Dtsch. Phys. Ges. 1917, 19, 82.

(85) Brillouin, L. J. Phys. 1926, 7, 353.

(86) Keller, J. Ann. Phys. 1958, 4, 180.

(87) Percival, I. C. J. Phys. B 1973, 6, L229.

(88) Percival, I. C. Adv. Chem. Phys. 1977, 36, 1.

(89) Mehta, M. Random Matrixes and the Statistical Theory of Energy Levels; Academic Press: New York, 1967.

(90) Ozorio de Almeida, A. Hamiltonian Systems: Chaos and Quantization; Cambridge University Press: Cambridge, 1988.

(91) Pechukas, P. J. Chem. Phys. 1972, 57, 5577.

(92) Jung, C.; Taylor, H. S.; Jacobson, M. P. J. Phys. Chem. A 2001, 105,681 .

(93) Siebrand, W.; Williams, D. F. J. Chem. Phys. 1968, 49, 1860.

(94) Henry, B. R. Acc. Chem. Res. 1977, 10, 207. 

183.

(95) Long, M. E.; Swofford, R. K.; Albrecht, A. C. Science 1976, 191 ,

(96) Burberry, M. S.; Albrecht, A. C. J. Chem. Phys. 1979, 71, 4631.

(97) Wallace, R. Chem. Phys. 1975, 11, 189.

(98) Wallace, R.; Wu, A. A. Chem. Phys. 1979, 39, 221.

(99) Sage, M. L. Chem. Phys. 1979, 83, 1455.

(100) Chandler, D. W.; Farneth, W. E.; Zare, R. N. J. Chem. Phys. 1982, $77,4447$.

(101) Jasinski, J. M.; Frisoli, J. K.; Moore, C. B. J. Chem. Phys. 1983 $79,1312$.

(102) Sumpter, B. G.; Thompson, D. L. J. Chem. Phys. 1985, 82, 4557.

(103) Butler, L. J.; Ticich, T. M.; Likar, M. D.; Crim, F. F. J. Chem. Phys. 1986, 85, 2331.

(104) Scherer, N. F.; Doany, F. E.; Zewail, A. H.; Perry, J. W. J. Chem. Phys. 1986, 84, 1932

(105) Jaffé, C.; Brumer, P. J. Chem. Phys. 1980, 73, 5646.

(106) Sibert, E. L.; Hynes, J. T.; Reinhardt, W. P. J. Chem. Phys. 1982, $77,3583$.

(107) Stamatiadis, S.; Farantos, S. C.; Keller, H.-M.; Schinke, R. Chem. Phys. Lett. 2001, 344, 565.

(108) Darling, B. T.; Dennison, D. M. Phys. Rev. 1940, 57, 128.

(109) Xiao, L.; Kellman, M. E. J. Chem. Phys. 1989, 90, 6086.

(110) Li, Z.; Xiao, L.; Kellman, M. E. J. Chem. Phys. 1990, 92, 2251

(111) Xiao, L.; Kellman, M. E. J. Chem. Phys. 1990, 93, 5805

(112) Kellman, M. E.; Xiao, L. J. Chem. Phys. 1990, 93, 5821.

(113) Svitak, J.; Li, Z.; Rose, J.; Kellman, M. E. J. Chem. Phys. 1995 102,4340 .

(114) Joyeux, M. Chem. Phys. 1994, 185, 263.

(115) Joyeux, M. J. Chem. Phys. 1995, 102, 2816.

(116) Joyeux, M. Chem. Phys. 1996, 203, 281.

(117) Joyeux, M.; Michaille, L. ACH-Models Chem. 1997, 134, 573.
(118) Prosmiti, R.; Farantos, S. C.; Guo, H. Chem. Phys. Lett. 1999 $311,241$.

(119) Suzuki, I. J. Mol. Spectrosc. 1968, 25, 479.

(120) Iachello, F.; Oss, S.; Lemus, R. J. Mol. Spectrosc. 1991, 146, 56

(121) Teffo, J. L.; Sulakshina, O. N.; Perevalov, V. I. J. Mol. Spectrosc. 1992, 156, 48.

(122) Bernath, P. F.; Dulick, M.; Field, R. W.; Hardwick, J. L. J. Mol. Spectrosc. 1981, 86, 275 .

(123) Vasudev, R. Chem. Phys. 1982, 64, 167

(124) Brasen, G.; Demtröder, W. J. Chem. Phys. 1999, 110, 11841.

(125) Pique, J. P.; Manners, J.; Sitja, G.; Joyeux, M. J. Chem. Phys. 1992, 96, 6495

(126) Quack, M. Annu. Rev. Phys. Chem. 1990, 41, 839.

(127) Lu, Z.; Kellman, M. E. J. Chem. Phys. 1997, 107, 1.

(128) Keshavamurthy, S.; Ezra, G. S. J. Chem. Phys. 1997, 107, 156

(129) McCoy, A. B.; Sibert, E. L. J. Chem. Phys. 1996, 105, 459.

(130) Jacobson, M. P.; Field, R. W. J. Phys. Chem. A 2000, 104, 3073

(131) Troellsch, A.; Temps, F. Z. Phys. Chem. 2001, 215, 207.

(132) Sibert, E. L.; McCoy, A. B. J. Chem. Phys. 1996, 105, 469.

(133) Jacobson, M. P.; Jung, C.; Taylor, H. S.; Field, R. W. J. Chem. Phys. 1999, 111, 600.

(134) Jung, C.; Ziemniak, E.; Taylor, H. S. J. Chem. Phys. 2001, 115 2499.

(135) Jacobson, M. P.; Child, M. S. J. Chem. Phys. 2001, 114, 250.

(136) Jacobson, M. P.; Child, M. S. J. Chem. Phys. 2001, 114, 262.

(137) Sievers, A. J.; Takeno, S. Phys. Rev. Lett. 1988, 61, 970.

(138) MacKay, R. S.; Aubry, S. Nonlinearity 1994, 7, 1623

(139) Aubry, S. Physica D 1997, 103, 201.

(140) Kopidakis, G.; Aubry, S. Physica D 1999, 130, 155.

(141) Kopidakis, G.; Aubry, S. Physica D 2000, 139, 247. 